\title{
A Mathematical Model for Infiltration Heat Recovery
}

\author{
C. R. Buchanan and M. H. Sherman ${ }^{1}$ \\ Energy Performance of Buildings Group \\ Indoor Environment Department \\ Environmental Energy Technologies Division \\ Lawrence Berkeley National Laboratory \\ University of California
}

\section{Abstract}

Infiltration has traditionally been assumed to affect the energy load of a building by an amount equal to the product of the infiltration flow rate and the sensible enthalpy difference between inside and outside. However, laboratory and simulation research has indicated that heat transfer between the infiltrating air and walls may be substantial, reducing the impact of infiltration. In this paper, two- and three-dimensional CFD simulations are used to study the fundamental physics of the infiltration heat recovery process and a simple macro-scale mathematical model for the prediction of a heat recovery factor is developed. CFD results were found to compare well (within about 10 percent) with limited published laboratory data corresponding to one of the scenarios examined. The model, based on the steady-state one-dimensional convection-diffusion equation, provides a simple analytical solution for the heat recovery factor and requires only three inputs: the infiltration rate, the Uvalue for the building, and estimates of the effective areas for infiltration and exfiltration. The most difficult aspect of using the model is estimation of the effective areas, which is done here through comparison with the CFD results. With proper input, the model gives predictions that agree well with CFD results over a large range of infiltration rates. Results show that infiltration heat recovery can be a substantial effect and that the traditional method may greatly over-predict the infiltration energy load, by 80-95 percent at low leakage rates and by about 20 percent at high leakage rates. This model for infiltration heat recovery could easily be incorporated into whole-building energy analysis programs to help provide improved predictions of the energy impact of infiltration.

\footnotetext{
${ }^{1}$ LBNL-44294: This work was supported by the Assistant Secretary for Energy Efficiency and Renewable Energy, Office of Building Technology of the U.S. Department of Energy under contract no. DE-AC0376 SF00098.
} 


\section{Table of Contents}

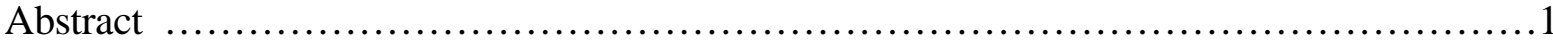

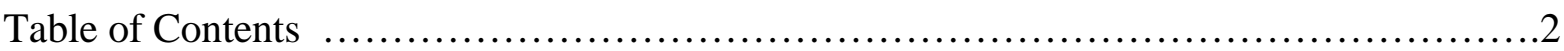

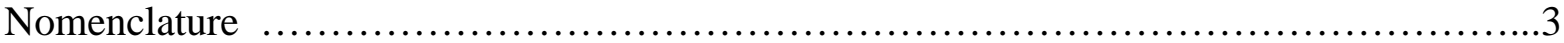

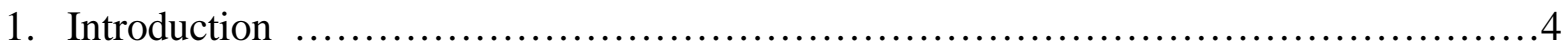

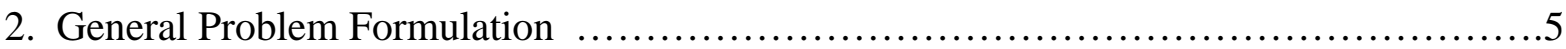

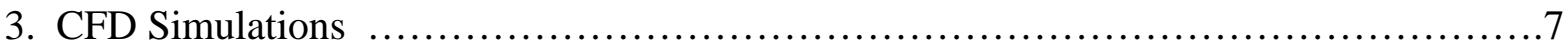

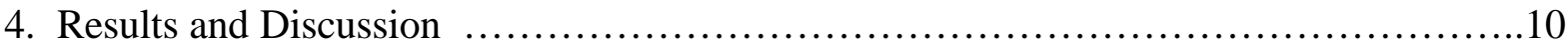

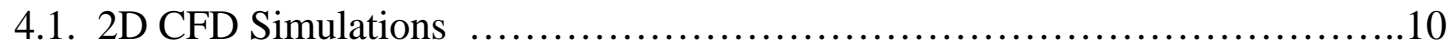

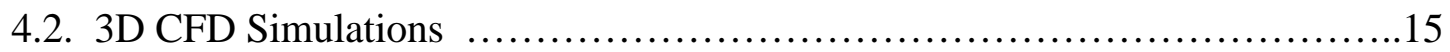

4.3. Experimental Comparison of CFD Results ............................. 17

5. Development of the Simplified Infiltration Heat Recovery Model .......................17

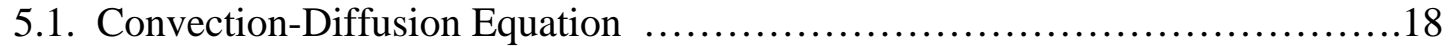

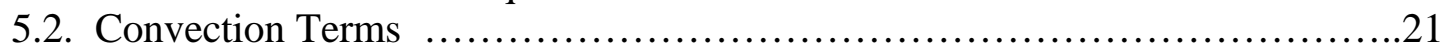

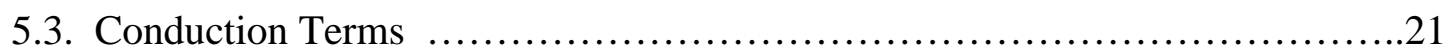

5.4. Introduction of a Dimensionless Flow Rate ...............................24

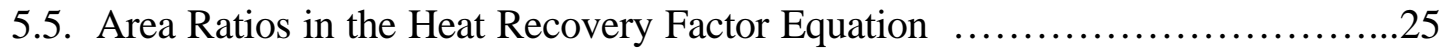

5.6. Plots of the Heat Recovery Factor .....................................25

5.4. Comparison of Mathematical Model with CFD Results . ...................27

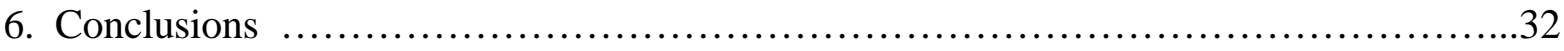

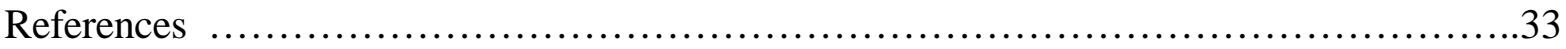

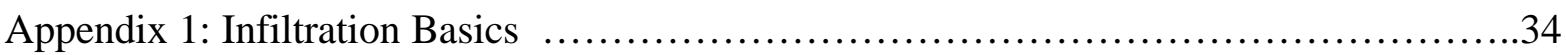

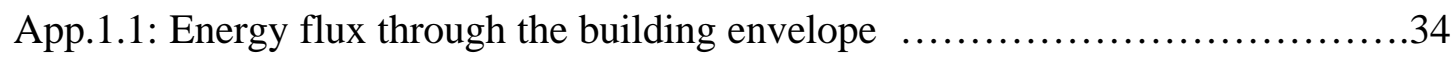

App.1.2: Conventional method of accounting for infiltration energy load ...........35

App.1.3: Including Infiltration Heat Recovery in the conventional method ..........35

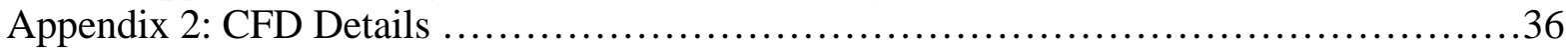

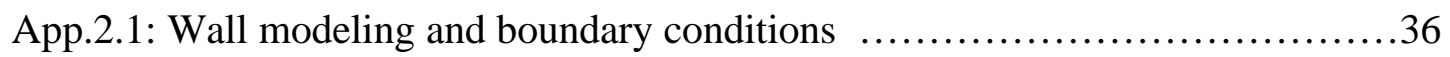

App.2.2: Thermal equilibrium in the wall insulation ................................ 38

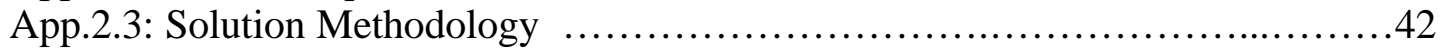

Appendix 3: Sample CFD Results: Energy Flux through Leaking Walls .................43

Appendix 4: A Worked Example Using the Infiltration Heat Recovery Factor ............47 


\section{Nomenclature}

$a_{i}=$ dimensionless flow rate (-)

$a_{o}=$ dimensionless flow rate based on total building surface area (-)

$A=$ building envelope total surface area $\left(\mathrm{m}^{2}\right)$

$A_{i}=$ effective areas for heat recovery model $\left(\mathrm{m}^{2}\right)$

$c_{p}=$ specific heat capacity of air $(1006 \mathrm{~J} / \mathrm{kg} \mathrm{K})$

$c_{p s}=$ specific heat capacity of insulation solid component $(1006 \mathrm{~J} / \mathrm{kgK})$

$c_{p w}=$ specific heat capacity of wall sheathing $(1200 \mathrm{~J} / \mathrm{kgK})$

$C_{1}, C_{2}, C_{3}=$ constants for heat recovery model variable area ratio expression (-)

$e=$ external wall faces for conduction terms in model development

$f_{l}=$ effective area ratio for infiltrating wall (-)

$f_{2}=$ effective area ratio for exfiltrating wall (-)

$g=$ gravity $\left(9.81 \mathrm{~m} / \mathrm{s}^{2}\right)$

$k=$ air thermal conductivity $(0.025 \mathrm{~W} / \mathrm{mK})$

$k_{\text {eff }}=$ effective thermal conductivity of insulation $(0.025 \mathrm{~W} / \mathrm{mK})$

$k_{s}=$ thermal conductivity of insulation solid component $(0.041 \mathrm{~W} / \mathrm{mK})$

$k_{w}=$ wall sheathing thermal conductivity $(0.13 \mathrm{~W} / \mathrm{mK})$

$L=$ wall thickness $(\mathrm{m})$

$m=$ infiltration mass flow rate $(\mathrm{kg} / \mathrm{s})$

$p=$ air pressure $(\mathrm{Pa})$

$\mathrm{Pe}=$ Peclet number $(-)$

$q_{o}=$ heat energy conducted through wall in model $\left(\mathrm{W} / \mathrm{m}^{2}\right)$

$Q=$ actual total (conduction and convection) building energy load (W)

$Q_{\text {cond }}=$ conduction energy flux through envelope in simplified model (W)

$Q_{\text {conv }}=$ convection energy flux through envelope in simplified model(W)

$Q_{\text {inf }}=$ actual energy load due to infiltration (W)

$Q_{\text {infC }}=$ conventional energy load due to infiltration $(\mathrm{W})$

$Q_{o}=$ pure conduction energy load with no infiltration $(\mathrm{W})$

$t=$ time $(\mathrm{s})$

$T=$ temperature $(\mathrm{K})$

$T_{i}=$ inside air temperature $(298 \mathrm{~K})$

$T_{o}=$ outside air temperature $(274 \mathrm{~K})$

$T_{s}=$ temperature of insulation solid component $(\mathrm{K})$

$T_{w}=$ wall sheathing temperature $(\mathrm{K})$

$u=$ air flow velocity $(\mathrm{m} / \mathrm{s})$

$U=$ wall $\mathrm{U}$-value $\left(\mathrm{W} / \mathrm{m}^{2}\right)$

$x=$ distance co-ordinate $(\mathrm{m})$

$\alpha=$ insulation permeability $\left(10^{-8} \mathrm{~m}^{2}\right)$

$\Delta T=T_{i}-T_{o}(24 \mathrm{~K})$

$\varepsilon=$ infiltration heat exchange effectiveness or heat recovery factor (-)

$\Gamma=$ generic diffusion coefficient $(\mathrm{kg} / \mathrm{m} \mathrm{s}$ in this paper)

$\phi=$ mass fraction of air in wall insulation material (0.99)

$\mu=\operatorname{air}$ viscosity $\left(1.72 \times 10^{-5} \mathrm{~kg} / \mathrm{ms}\right)$

$\rho=$ air density $\left(\mathrm{kg} / \mathrm{m}^{3}\right)$

$\rho_{s}=$ density of insulation solid component $\left(70 \mathrm{~kg} / \mathrm{m}^{3}\right)$

$\rho_{w}=$ wall sheathing density $\left(544 \mathrm{~kg} / \mathrm{m}^{3}\right)$ 
$\tau=$ fluid stress tensor $\left(\mathrm{N} / \mathrm{m}^{2}\right)$

\section{Introduction}

Infiltration, accidental air leakage through building envelopes, is a common phenomenon that affects both indoor air quality and building energy consumption. Infiltration can contribute significantly to the overall heating or cooling load of a building, but the magnitude of the effect depends on a host of factors, including environmental conditions, building design and operation, and construction quality. Claridge and Bhattacharyya (8) note that a great deal of work has been devoted to the prediction and measurement of infiltration rates in building systems, but little effort has been directed toward determining the actual energy impact of infiltration.

Few studies regarding the energy issues of infiltration have been found in the literature. Based on field measurements taken at 50 residential buildings, Caffey (6) concluded that up to 40 percent of the heating/cooling costs in the homes studied was due to infiltration. In another study of residential buildings, Persily (15) attributed about one-third of the heating/cooling requirements to infiltration. Sherman and Matson (16) examined measured leakage data and found that a high fraction of the space conditioning load in U.S. residential buildings was due to infiltration. The results of a recent study (14) of U.S. office buildings performed by the National Institute of Standards and Technology (NIST) estimates that air leakage accounts for about 15 percent of the heating load in office buildings nationwide and about 1 or 2 percent of the cooling load. By all measures, the impact of infiltration can be sizeable and should therefore be considered in calculations of building energy consumption.

$$
Q_{\text {infC }}=m c_{p}\left(T_{i}-T_{o}\right)
$$

The conventional method of accounting for the extra load due to infiltration (explained in Appendix 1) is to add a simple convective transport term of the form $m c_{p} T$ to the energy balance for the building. For single-zone building models the conventional infiltration load, $Q_{\text {infC, }}$, shown in equation 1 , is the product of the infiltrating air mass flow rate, the specific heat capacity of air, and the temperature difference between inside and outside. This relation does not include the effects of moisture in the air and is strictly valid only if the leaking air does not interact thermally with the building walls. In reality, leaking air exchanges heat with the walls as it enters and leaves the building, which changes the thermal profile in the walls and warms or cools the infiltrating/exfiltrating air. This results in different values for the conduction, infiltration, and total heat losses than are predicted by the conventional method (see Appendix 1). Some studies have shown that this effect could be substantial suggesting that the conventional method over-predicts the energy impact of infiltration $(2,4,7,8,11)$.

An improved prediction of the energy load due to infiltration can be made by introducing a correction factor, the infiltration heat exchange effectiveness, $\varepsilon$, or the heat recovery factor (defined by equation 2 ), into the expression for the conventional load (equation 1). In equation $2, Q$ is the actual total energy load of the building with infiltration and $Q_{o}$ is the conduction load when there is no infiltration. This heat recovery factor, introduced by Claridge and Bhattacharyya (8), accounts for the thermal interaction between 
leaking air and building walls. The actual infiltration load, $Q_{\text {inf }}$, is calculated using the heat recovery factor as shown in equation 3 (detailed in Appendix 1).

$$
\begin{gathered}
\varepsilon \equiv 1-\frac{Q-Q_{o}}{m c_{p} \Delta T}=1-\frac{Q-Q_{o}}{Q_{\text {inf } C}} \\
Q_{\text {inf }}=(1-\varepsilon) m c_{p}\left(T_{i}-T_{o}\right)=(1-\varepsilon) Q_{\inf C}
\end{gathered}
$$

At this point, basic information regarding the physical details of the problem (like general air flow structure or important transport mechanisms) or the importance of certain variables (like wall design, leakage path, bulk air temperature difference) is not available, therefore, infiltration heat recovery is not well understood. The purpose of this study is to investigate the heat transfer process between infiltrating air and room walls and determine its effect on the energy load conventionally attributed to infiltration. A primary goal of this work is to provide a foundation of knowledge about this process by which a fundamental understanding can be developed and a direction for future work can be determined. Another goal is to determine the rough size of the infiltration heat recovery effect for a variety of leakage scenarios. If the effect is not sizeable, then there would be no point in further work. A final goal is to develop a simplified mathematical model for calculation of the infiltration heat recovery factor that is based on the important physics of the process.

In this paper, two- and three-dimensional computational fluid dynamics (CFD) simulations are used to investigate the basic physics of the infiltration heat recovery process. We choose to start with a fairly simple physical representation (only conduction and convection are considered for transport) so that an understanding of the phenomenon can be developed from first principles. Additional processes, like turbulence or radiation, can be added progressively if necessary. Also, a one-dimensional mathematical model is developed that can be used to determine the extent of heat transfer between leaking air and walls, represented quantitatively as the infiltration heat recovery factor. This macro-scale model, based on the steady-state one-dimensional convection-diffusion equation, provides a simple analytical relation for the heat recovery factor. It requires only three inputs: the infiltration rate, the U-value for the building, and estimates of the effective areas for infiltration and exfiltration. Predictions from the model are compared with results from detailed CFD simulations and limited experimental results from the literature.

\section{General Problem Formulation}

The cross-section of a hypothetical test room under a general infiltration scenario is shown in figure 1. Small holes in the outer sheathing of the building envelope (plywood in this study) allow air to leak into the wall cavity and flow through the wall from outside to inside for the infiltrating wall and vice-versa for the exfiltrating wall. The driving force for leakage is a pressure differential due to wind and temperature differences between inside and outside. Figure 1 shows the infiltration problem in its entirety, but in this study only a limited portion of this environment (the walls and the airspace in the vicinity of the walls) is analyzed 
to help understand the infiltration heat recovery process. As infiltration heat recovery is largely a localized process this is all that is necessary.

Eight wall configurations, shown in figures 2 and 3, are examined under various environmental conditions. Wall geometries 1,2, 5, and 8 have insulation in the full wall cavity, while geometries 3 and 4 have empty wall cavities. Walls 6 and 7 are special cases with only half of the wall cavity insulated. Walls 1, 3, 6, and 7 provide a long flow path for infiltrating air that could potentially create a displacement flow in the wall cavity. Walls 2, 4, and 5 provide a short flow path for infiltrating air that could isolate air in the top and bottom sections of the wall. Wall 8 has the possibility for both long and short paths. Leakage rates through the wall are varied and the inside/outside temperature difference is fixed at $24 \mathrm{~K}$ for most of the cases. The influence of the inside/outside temperature difference is also examined.

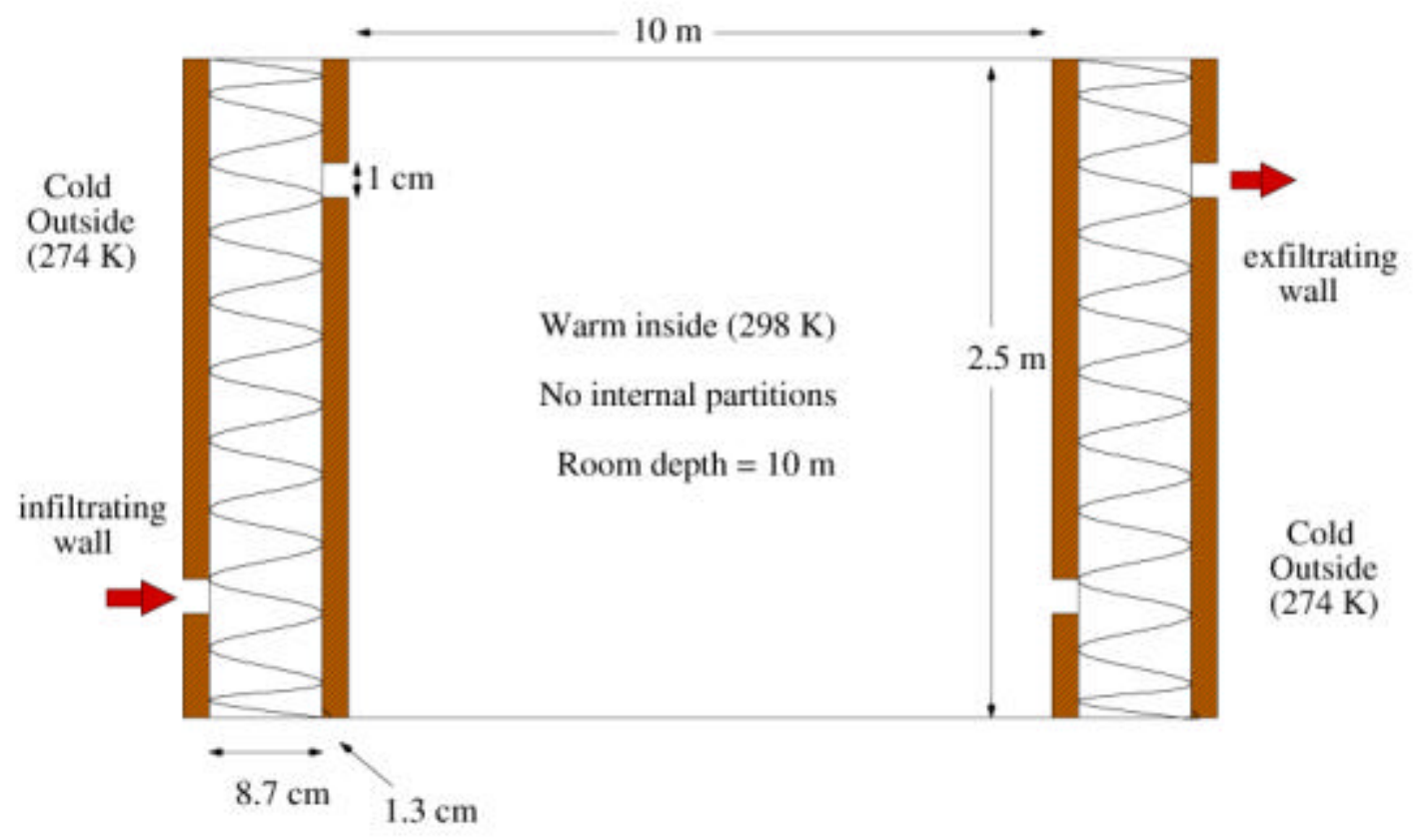

Figure 1: Cross-section of a hypothetical test room showing the general infiltration problem (wall geometry 1 shown). The infiltrating and exfiltrating walls have a conduction and convection energy flux, but all other walls have only a conduction flux.

The example room shown in figure 1 could represent a row-house inner unit and is composed of a ceiling, floor, front wall, and rear wall with no air leakage and an infiltrating wall with a corresponding exfiltrating wall both with air leakage. Heat conduction occurs through all of the walls but the infiltrating and exfiltrating walls also have convective heat transfer.

We believe the important physics of the infiltration heat recovery process occurs largely within the wall structure and in the vicinity of the wall surfaces (i.e., a few centimeters) and the results support this notion. Since the purpose of this study is to understand the important physics of heat recovery, it is only necessary to analyze this select region. It is not necessary to represent the details of the building interior or the entire building envelope, therefore, the room interior is not represented and the building envelope is 
separated into non-interacting wall elements, which are examined individually. Information from the individual walls is added together to determine the overall impact for a complete room system. The leaking walls are both of the same geometry type and are matched by their air leakage rates. The bulk air flow within the room is not represented, but this should not be a problem because, as Etheridge (9) notes, the internal room air flow has only a secondary effect on infiltration. The most important influences on infiltration are wind-induced pressure differences and buoyancy of room air in the vicinity of the wall. It is possible that radiation has some effect on the heat recovery process, but this topic is not examined here. The importance of radiation will be assessed in future work.

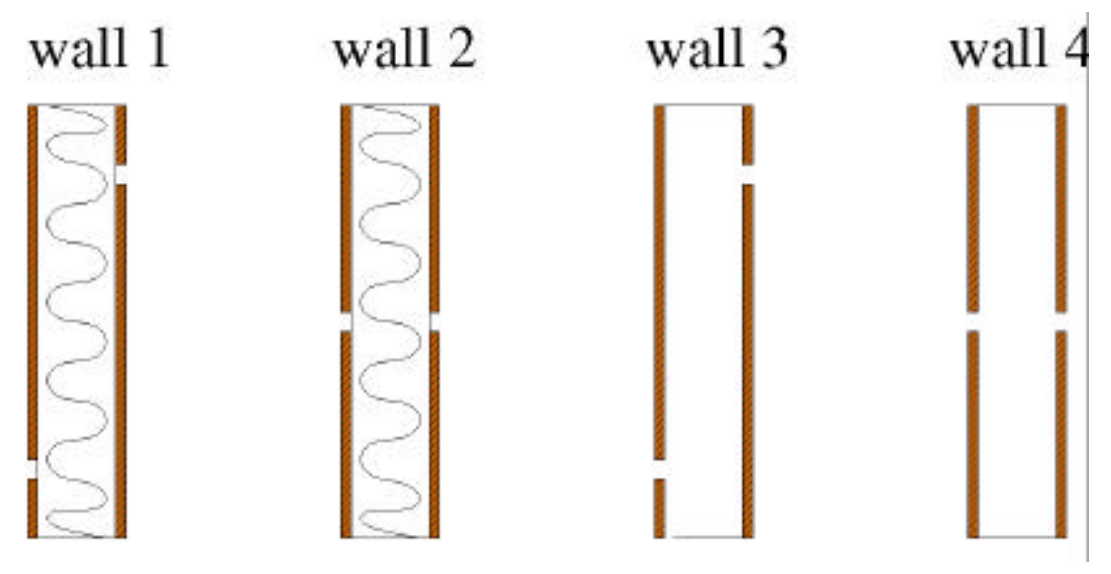

Figure 2: Wall geometries $1-4 ; 1 \& 2$ are insulated and $3 \& 4$ are empty.

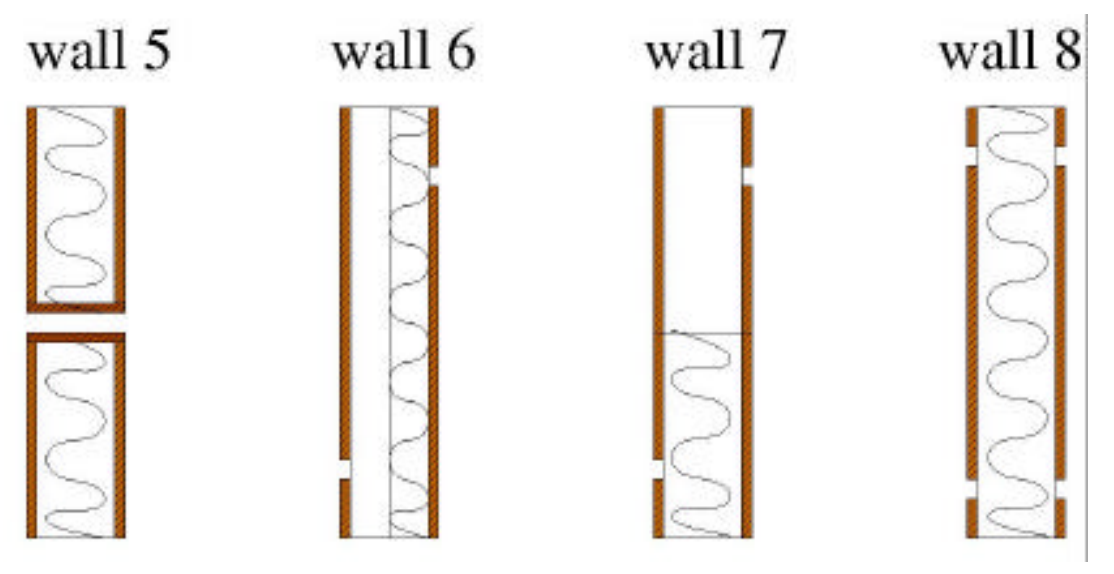

Figure 3: Wall geometries 5-8; special cases.

\section{CFD Simulations}

The purpose of this study is to investigate the potential heat transfer between infiltrating air and room walls, quantify its extent through a heat recovery factor, and determine how this process could affect the energy load conventionally attributed to infiltration. Previous studies have measured lump quantities experimentally $(2,7,8)$ or 
simulated the system using simplified modeling, for example, a prescribed Nusselt number for the air-wall heat transfer (11). Despite prior research efforts, the fundamental details of the process are still not known and, therefore, it is not well understood.

In the first part of this study, computational fluid dynamics (CFD) simulations are used to examine the basic physics of the infiltration heat recovery process in detail. The individual contributions of conduction and convection to the total heating load are determined without making fundamental simplifications as in previous work. These components of the total energy flux are used to calculate the infiltration heat recovery factor.

An advantage of CFD simulations over experimental studies is that details of the system, like local flow patterns and thermal profiles, can be resolved helping to provide a better overall understanding of the physics involved. Also, many important aspects of the problem that are often difficult to measure, like boundary conditions, material properties, and flow paths, are prescribed and can be systematically varied with ease. This makes correlations between different variables, like leakage path length and heat recovery, much easier. Also, these simulations are quick and inexpensive compared to experiments and can be used to help design experiments. A disadvantage of such simulations is that it is difficult to represent the complexity of the true system, especially the variations associated with construction quality and material properties. The systems here are idealized, having homogeneous material properties and ideal construction. However, the results should be representative and of practical value with proper interpretation.

The walls are modeled as two- and three-dimensional systems in the CFD simulations. Flow and energy transport in the air are determined via the Navier-Stokes and energy equations, equations 4-6, respectively. A laminar representation is used for the flow, and solutions show this to be a valid assumption, as the highest calculated Reynolds number inside or near the wall is only about 2000 , based on wall thickness. It is possible that turbulence could have some effect even at these moderately low Reynolds numbers, so this will be examined in future work. The plywood sheathing is represented as an impermeable, solid material. Energy transport within the sheathing is calculated via the conduction equation, shown in equation 7. Insulation, if present in the wall, is represented as a porous material. Air flow through the insulation is determined via Darcy's Law, equation 8, a common model for flow through porous media $(5,11)$. Energy transport through the insulation is determined via a modified form of the energy equation, as given by equation 9 . In equation 9, an effective conductivity, given by equation 10, is used in the conduction flux term and the thermal inertia of the solid component is included in the transient term. A fundamental assumption in the validity of equation 9 for this application is local thermal equilibrium between the fluid and solid phases in the porous media. This assumption and other details of the wall modeling are discussed in Appendix 2.

$$
\begin{gathered}
\frac{\partial \rho}{\partial t}+\frac{\partial \rho u_{i}}{\partial x_{i}}=0 \\
\frac{\partial \rho u_{i}}{\partial t}+\frac{\partial \rho u_{i} u_{j}}{\partial x_{j}}=-\frac{\partial p}{\partial x_{i}}+\frac{\partial \tau_{i j}}{\partial x_{j}}+\rho g_{i}
\end{gathered}
$$




$$
\begin{gathered}
c_{p} \frac{\partial \rho T}{\partial t}+c_{p} \frac{\partial \rho u_{i} T}{\partial x_{i}}=k \frac{\partial^{2} T}{\partial x_{i}{ }^{2}}+\frac{\partial p}{\partial t}+u_{i} \frac{\partial p}{\partial x_{i}} \\
\rho_{w} c_{p w} \frac{\partial T_{w}}{\partial t}=k_{w} \frac{\partial^{2} T_{w}}{\partial x_{i}{ }^{2}} \\
\frac{\partial \rho u_{i}}{\partial t}=-\frac{\partial p}{\partial x_{i}}+\frac{\mu}{\alpha} u_{i}+\rho g_{i} \\
\frac{\partial}{\partial t}\left(\phi c_{p} \rho T+(1-\phi) c_{p s} \rho_{s} T\right)+c_{p} \frac{\partial \rho u_{i} T}{\partial x_{i}}=k_{e f f} \frac{\partial^{2} T}{\partial x_{i}{ }^{2}}+\frac{\partial p}{\partial t}+u_{i} \frac{\partial p}{\partial x_{i}} \\
k_{e f f}=\phi k+(1-\phi) k_{s}
\end{gathered}
$$

Thermal gradients in the system develop due to the difference between indoor and outdoor conditions and give rise to natural convection. As mentioned previously, it is important to represent the effects of buoyancy on the flow to properly determine infiltration rates and the heat flux at the wall due to boundary layer formation, so buoyancy is included in these simulations. A temperature-dependent empirical equation of state for the fluid density, coupled with the body force term in the fluid momentum equation in the vertical direction introduces the effects of buoyancy into the flow.

Simulations are performed for the eight wall geometries shown in figures 2 and 3 under various infiltration rates with a constant temperature difference of $24 \mathrm{~K}$ between inside and outside. Air leakage is achieved by imposing either velocity or pressure boundary conditions at locations far enough from the wall construction to prevent local disturbances from developing near the wall. This requires there to be some amount of empty space on either side of the wall. An empty airspace of about twice the wall thickness is placed on either side of the wall, which allows a natural convection boundary layer to develop.

Temperature boundary conditions are imposed upon the air on either side of the wall, not upon the wall itself, meaning heat transfer through the wall structure is calculated entirely as conjugate heat transfer, i.e., no assumptions are made about the heat transfer (see Appendix 2 for details).

Due to the complexity of the flow, it is not possible to achieve a converged solution, based on the sum of the normalized residuals, using the steady-state equations. Therefore, the time-dependent equations are integrated in time until steady-state is reached. The details of the solution methodology are discussed in Appendix 2. Comparison of results from twodimensional simulations using a coarse computational grid (33,000 nodes) and a fine grid (140,000 nodes) for the same wall geometry show that the coarse grid is sufficient to provide a grid-independent solution. All two-dimensional results presented here are from converged steady-state solutions using a 33,000-node grid. In the three-dimensional simulations, a different grid with approximately 100,000 nodes is used. Grid-dependency tests are not performed for the three-dimensional cases because the required computational resources are not available. However, it can be inferred from the corresponding two-dimensional cases that this grid resolution should be roughly sufficient to provide some useful results. Examination of the two-dimensional results shows that about 5 to 10 grid points across the thermal and 
momentum boundary layers is enough to provide a grid independent solution. In the threedimensional simulations, about 5 grid points are used across the boundary layers, which appears to provide fairly good resolution. Although the three-dimensional results look good and are from converged solutions, it is not known if the solutions are grid independent. This should be kept in mind when examining the three-dimensional results. All three-dimensional results are from converged steady-state solutions using a grid with approximately 100,000 nodes.

\section{Results and Discussion}

The main point of interest is the extra energy load introduced by infiltration because this will allow calculation of the heat recovery factor. This is determined by first calculating the heat flux through the room walls with no air leakage, designated as $Q_{o}$. Then, the energy flux is determined for the same wall types with air leakage. The difference between the two values is the infiltration-induced energy load. The convection and conduction energy flux across the external (outside) face of each wall is calculated for infiltrating and exfiltrating configurations. Using the external building face for the system control volume boundary is an arbitrary choice. The interior face could be used as well, however, it is important from an organizational standpoint that the energy accounting be performed at a consistent location. Detailed results from the wall 1 configuration are shown in Appendix 3 to illustrate the trends that occur in the flux components as the leakage rate varies. Also, an example is worked in Appendix 4 using the wall 1 results to illustrate how the heat recovery information can be used and the potential increases in accuracy it could provide for building energy load predictions.

\subsection{D CFD Simulations}

Figure 4 shows the heat recovery factor for wall geometries 1-4 determined from twodimensional CFD simulations. The variable on the horizontal axis of the graph is the dimensionless flow rate $\left(a_{o}\right)$, defined in equation 11. It is the leakage rate nondimensionalized by the U-value of the building and the specific heat of air. It was found to be a useful independent variable when comparing the heat recovery for different cases because it collapses the data showing the universal trends. In all cases, the heat recovery factor approaches a value of one at very low flow rates and decreases with increasing flow rate. Heat transfer is lower at high flow rates because there is less time for energy to be transported from the walls to the infiltrating air resulting in lower heat recovery.

$$
a_{i}=\frac{m c_{p}}{U_{i} A_{i}}
$$

Two distinct trends can be seen in figure 4. One trend is that the walls with holes in a high/low configuration, walls 1 and 3, have a significantly higher heat recovery factor than the walls with holes that are straight through, but these straight through geometries still have a significant heat recovery effect. This is partly because the high/low configuration has a longer leakage path and, for a given flow rate, the air remains within the wall cavity for a longer period of time. This allows for greater heat transfer and higher heat recovery compared to the straight through case. 
The other trend is that data points for the high/low configurations fall roughly on a single trend line, and the same is true for the straight through configurations. That is, insulated ( $1 \& 2)$ and empty walls ( $3 \& 4)$ with the same hole configuration have about the same heat recovery when plotted against the chosen independent variable, $a_{o}$. This suggests a universal behavior that may be applicable to all leakage scenarios. Note that the insulated and empty walls have different leakage rates for a given value of $a_{o}$ because their U-values are different, but they have about the same heat recovery. This indicates that the nondimensional flow rate, $a_{o}$, is the proper independent variable to use when comparing different cases.

The heat recovery factor is calculated for wall 5 using four different flow rates and is compared to the data for wall 2 in figure 5 . The geometry of wall 5 is the same as wall 2 , except the hole in the sheathing continues all the way through the wall, including the insulation, and the leaking air is separated from the insulation by a layer of the sheathing material. Interestingly, this change in geometry has little effect on the heat recovery. The four points calculated for wall 5 fall essentially on the same trend line as the points for wall 2 . This suggests that the interior details of the leakage path do not have a great affect on the heat recovery, just the overall hole geometry is important.

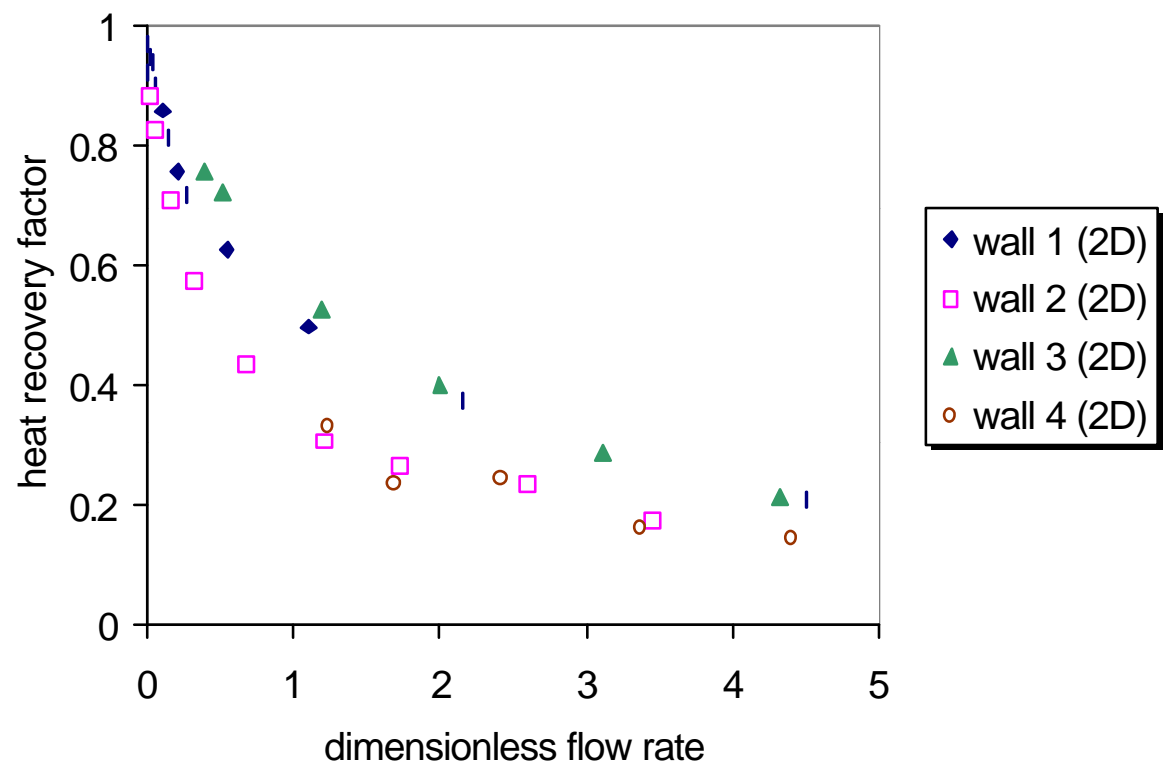

Figure 4: Heat recovery factor determined from 2D CFD simulations for walls 1-4. Solid symbols show data for walls with a high/low hole configuration (1 and 3) and hollow symbols show data for walls with a straight through hole configuration ( 2 and 4$)$. Notice the two distinct trends - one for each hole configuration. 


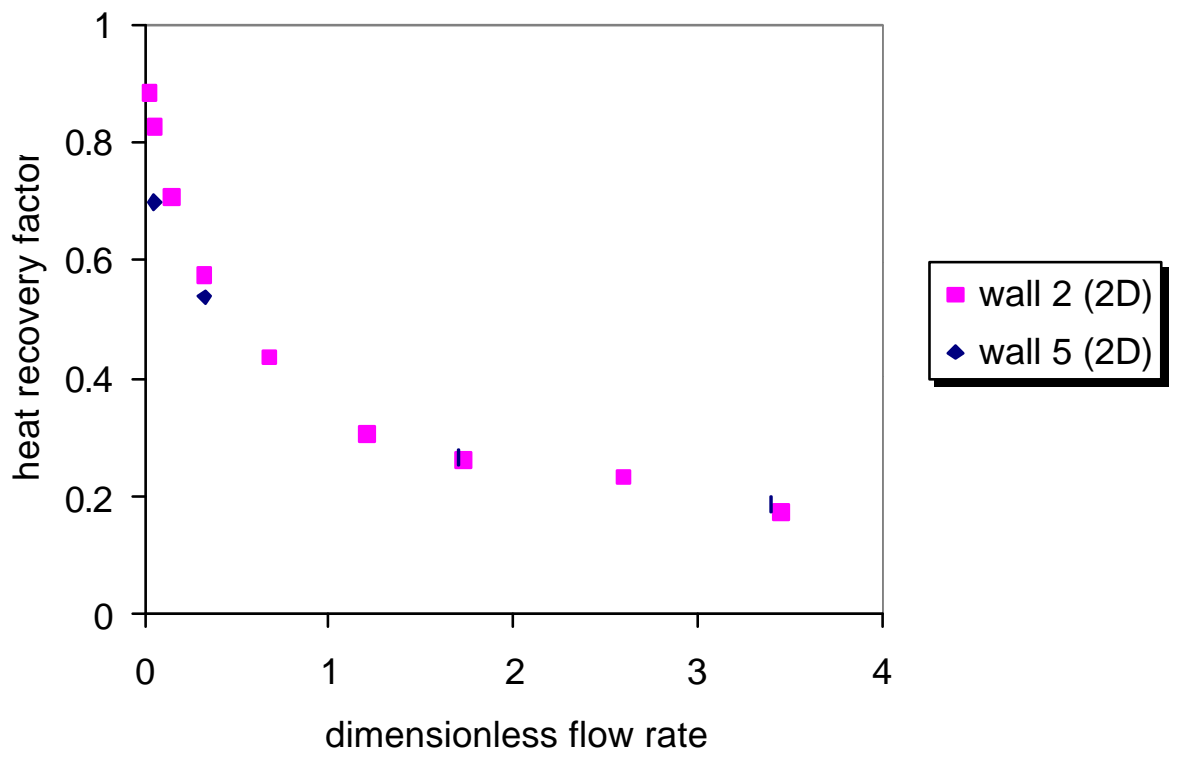

Figure 5: Heat recovery factor determined from 2D CFD simulations for walls 2 and 5. Both walls have a straight through hole configuration but the details of the leakage path are different-- see figures 2 and 3. Both walls have about the same heat recovery.

The heat recovery factor is calculated for walls 6 and 7 using several different flow rates and is compared to the data for wall 1 in figure 6 . The leakage path is the same for all of the walls, a high/low hole configuration, but the layout of the insulation in the wall cavity is different. We thought that this might have some impact on the heat recovery, so these special cases were investigated. As figures 2 and 3 show, the insulation fills the entire cavity of wall 1, while only half the cavity is filled with walls 6 and 7. Wall 6 has insulation on the right half of the cavity and wall 7 has insulation on the bottom half. As figure 6 shows, this change in the wall cavity insulation layout has no significant effect on the heat recovery. The points calculated for walls 1,6 , and 7 all fall on the same trend line. 


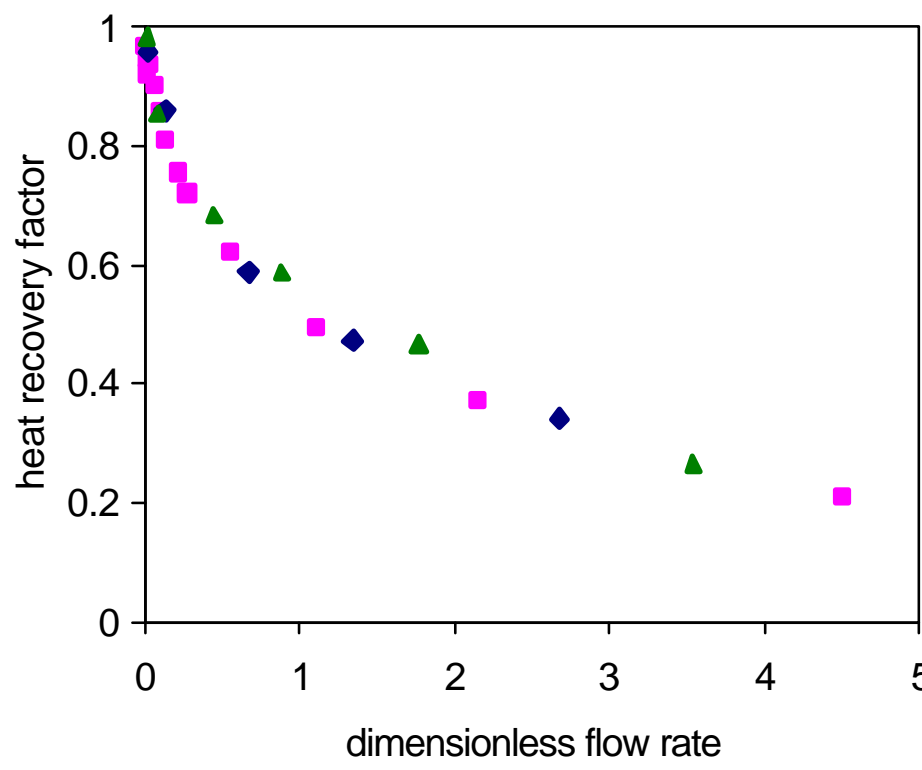

wall $1(2 \mathrm{D})$

- wall 6 (2D)

$\triangle$ wall $7(2 \mathrm{D})$

Figure 6: Heat recovery factor determined from 2D CFD simulations for walls 1, 6, and 7. All walls have a high/low hole configuration but the layout of insulation in the wall cavity is different for each case-- see figures 2 and 3. The heat recovery factor for all the walls follows a single trend line.

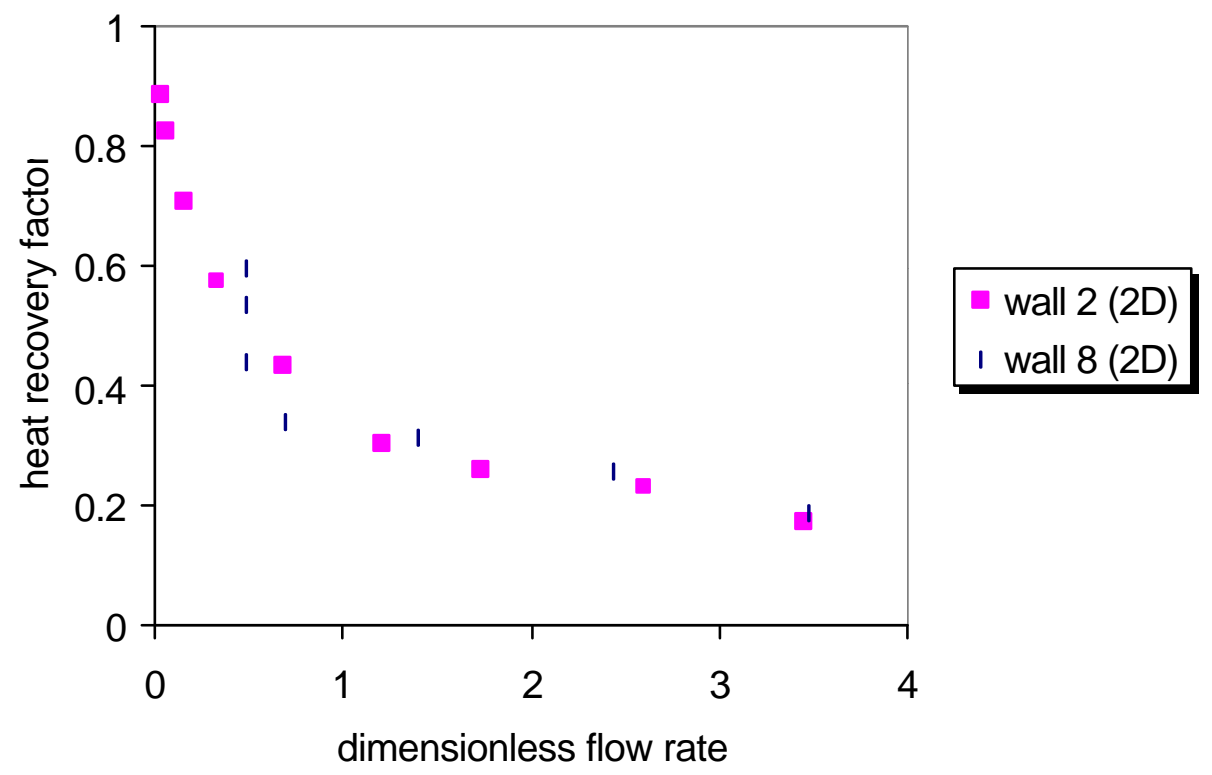

Figure 7: Heat recovery factor determined from 2D CFD simulations for walls 2 and 8 . Both walls have insulated wall cavities. Wall 2 has a single straight through hole configuration and wall 8 has two straight through holes-- see figures 2 and 3. 
The heat recovery data for walls 2 and 8 is shown in figure 7 . Wall 8 is similar to wall 2, having two straight through holes, but is also similar to wall 1 with two high/low holes. The heat recovery for wall 8 is closer to that of wall 2, however, suggesting that this configuration behaves more like two straight through holes than two high/low holes.

For values of $a_{o}$ less than one, the heat recovery factor for wall 8 shows strange behavior, dropping sharply with small changes in flow rate and seemingly not approaching one for no leakage. This strange behavior is due to two-way flow occurring in one or both of the walls caused by buoyancy induced pressures that act in different directions at different holes. For example, in a given wall air may flow into the room through the top hole and out of the room through the bottom hole. This allows multiple possibilities for flows at individual holes for a given overall leakage rate or $a_{o}$. The result is that for a given overall leakage rate there is not a unique heat recovery factor. At larger leakage rates higher driving pressures force the flow direction to be the same for both sets of holes and the data points for wall 8 show the same trend as wall 2. There is little interaction between the two sets of holes, i.e., the high set and low set, because the insulation separating them provides a large flow resistance. A wall of this design may not need to be modeled in its entirety. However, preliminary studies of this wall with an empty cavity show that there is a significant amount of interaction between the high and low holes, so this may not be a universal trait for all such wall designs. This wall geometry shows the potential complexity of real life situations and deserves further investigation.

A final point of interest is the influence of the bulk air temperature difference $\left(T_{i}-T_{o}\right)$ on the heat recovery factor as it may not scale directly with this temperature difference. CFD simulations were performed over a range of leakage rates to examine this possibility. The bulk air temperature difference was changed from $24 \mathrm{~K}$ to $12 \mathrm{~K}$ and $-18 \mathrm{~K}$ for the insulated wall with a high/low hole configuration (wall 1). The heat recovery factors for these cases are compared in figure 8 over the range of leakage rates. All three cases, with the $24 \mathrm{~K}, 12$ $\mathrm{K}$, and $-18 \mathrm{~K}$ difference, have essentially the same heat recovery values and trends. It seems that the heat recovery scales with the bulk air temperature difference, so this parameter can be removed from the analysis. 


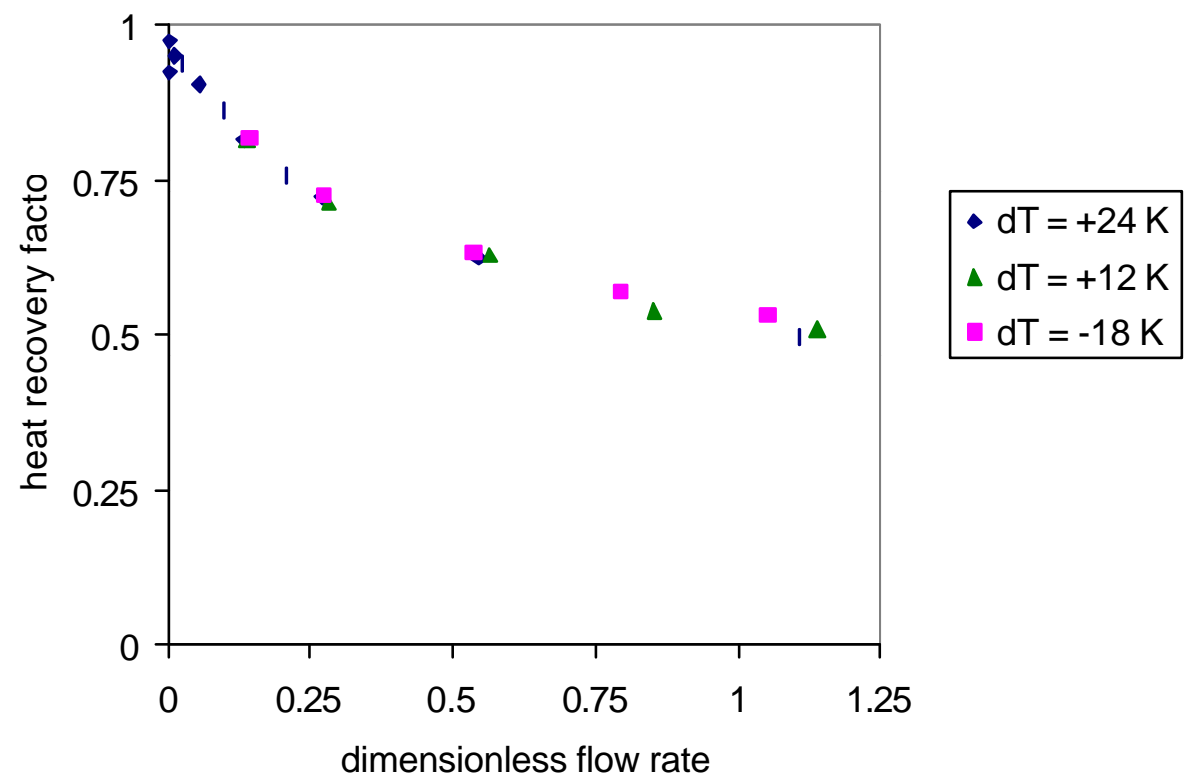

Figure 8: Heat recovery factor for wall 1 (insulated with a high/low hole configuration) determined from 2D CFD simulations using bulk air temperature differences $\left(T_{i}-T_{o}\right)$ of +24 $\mathrm{K},+12 \mathrm{~K}$ and $-18 \mathrm{~K}$. The bulk air temperature difference appears to have no affect on the heat recovery. Note the decreased range in the dimensionless flow rate.

\subsection{D CFD Simulations}

Three-dimensional simulations are performed for walls 1 and 2 and the results are compared to those from two-dimensional simulations in figures 9 and 10. In the 3D simulations, the hole in the wall sheathing is roughly a square, while the hole in the 2D cases corresponds to a long slit spanning the width of the wall. In all other respects, the $2 \mathrm{D}$ and $3 \mathrm{D}$ walls are geometrically the same. This geometric discrepancy causes differences in the air flow patterns in and around the wall, but it does not appear to have any significant effect on the heat recovery. The 3D simulations give nearly the same values and show almost the same trends for the heat recovery factor for a given hole configuration as the 2D simulations. Therefore, it may be sufficient to use 2D simulations to study a given wall geometry, which would mean a large savings in time and effort compared to 3D simulations. 


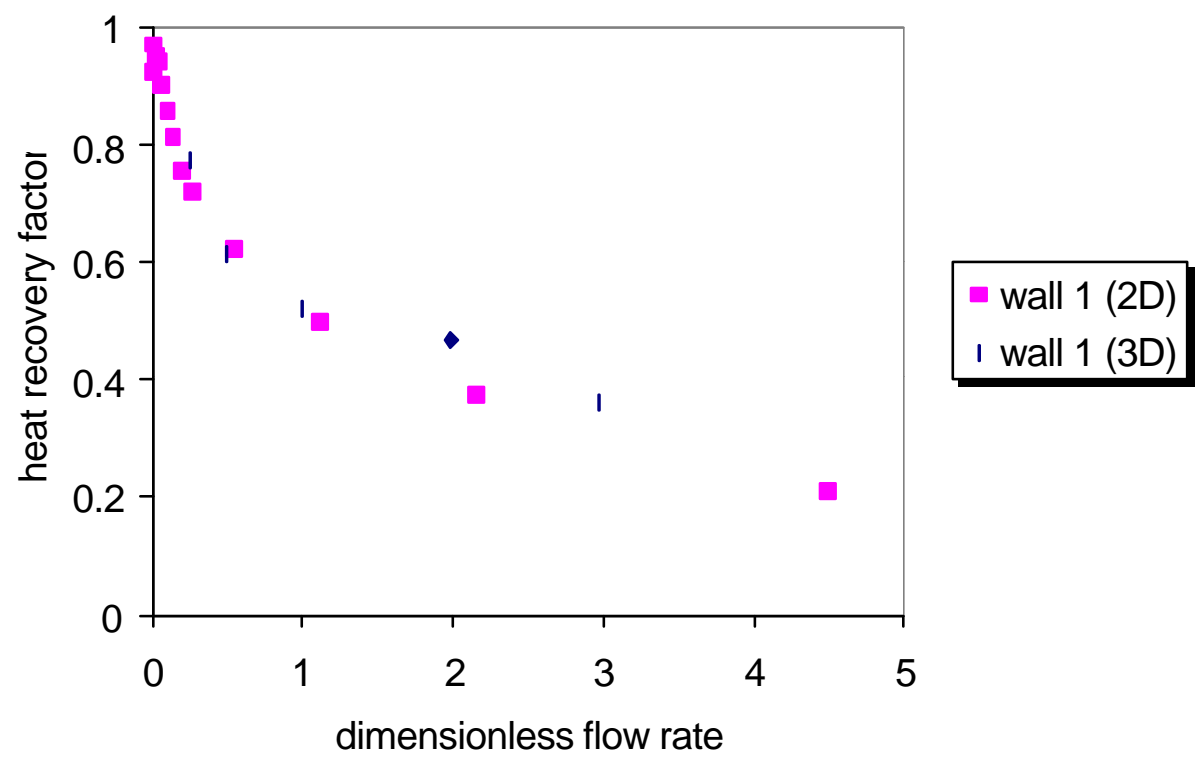

Figure 9: Heat recovery factor determined from 2D and 3D CFD simulations for wall 1, an insulated wall with a high/low hole configuration. Heat recovery values are essentially the same for both cases.

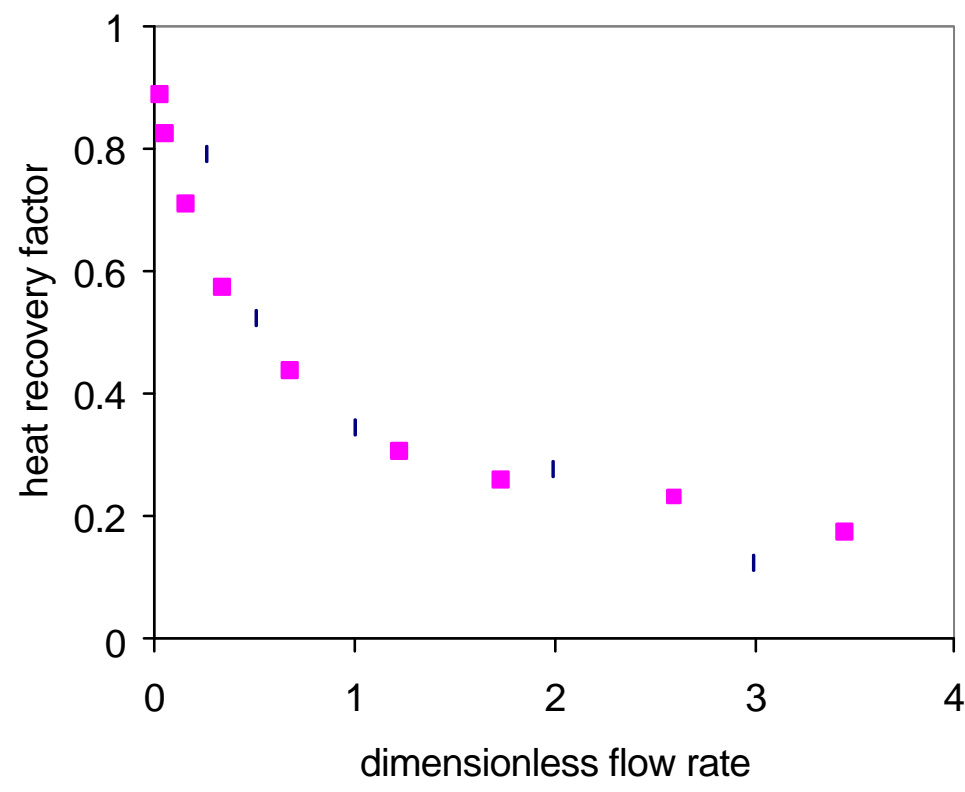

wall $2(2 \mathrm{D})$

I wall $2(3 \mathrm{D})$

Figure 10: Heat recovery factor determined from 2D and 3D CFD simulations for wall 2 , an insulated wall with a straight through hole configuration. Heat recovery values are essentially the same for both cases. 


\subsection{Experimental Comparison of CFD Results}

The only experimental data available for comparison is that of Claridge and Bhattacharyya (8). Unfortunately, their experiments do not correspond exactly to the work in this study, some important details of the experiments are not given making direct comparisons difficult, and leakage rates were varied only over a narrow range. However, one of their cases corresponds fairly well to one of ours and can be used for comparison.

The case of Claridge and Bhattacharyya with a diffuse inlet and outlet (inlet B4, outlet A) is similar to walls 1 and 3 in this study (high/low hole configuration). They calculated a heat recovery factor of about 0.80 when $a_{o}$ is 0.05 and about 0.65 when $a_{o}$ is 0.25 . As figure 8 shows, CFD results for wall 1 give a heat recovery factor of 0.90 when $a_{o}$ is 0.05 and about 0.72 when $a_{o}$ is 0.25 . There are no CFD data available for wall 3 at these low flow rates, but the trend is the same. Considering the rough nature of the comparison, the experimental and CFD results show good agreement. There is only about a 10 percent discrepancy in the heat recovery factor at both points. This suggests that the CFD results are qualitatively, and, most likely, quantitatively accurate. Also, this suggests that processes which occur naturally in the experiment but that are not represented in the simulations (turbulence, radiation) may not have a strong effect on the heat recovery. The CFD technique can, therefore, be considered a useful tool for study of the infiltration heat recovery process. The CFD results should provide a sound basis for comparison with our simplified infiltration heat recovery model.

\section{Development of the Simplified Infiltration Heat Recovery Model}

The objective of the following work is to develop a simple macro-scale mathematical model for the infiltration heat recovery factor, which is based on the important physics of the process. The model should provide a simple, yet accurate, means for calculating the heat recovery factor, $\varepsilon$, under a variety of environmental conditions, building designs, and leakage scenarios. The starting point is the steady-state one-dimensional convection-diffusion equation (17), shown in equation 12. This simple representation is used because we believe it includes the most important physical mechanisms and will help provide insight into the heat recovery process more easily than a complex representation. It is a simplified form of the general transport equation, which appeared earlier in the CFD simulations as the NavierStokes and energy equations (equations 5,6, and 9). In this case, the transient and source terms are not included and only one dimension is considered.

The immediate purpose of this model is to give a rough idea of the size of the infiltration heat recovery effect, not incorporation into network codes for dynamic building simulation. If the effect is sizeable and the topic merits further investigation, additions can be made to the model in future work, if necessary, to help provide more accurate, realistic results and to make it suitable for use in network codes. 


\subsection{Convection-Diffusion Equation}

The one-dimensional convection-diffusion equation below represents transport by combined convection and diffusion in a steady flow varying in one spatial direction, i.e., a one-dimensional flow. This relation is valid for flows existing in three-dimensional space (all real flows), but only those that vary in one direction, for example, a fully-developed pipe flow. In equation 12, $\phi$ represents any scalar flow variable, e.g., temperature or concentration, and $\Gamma$ is the diffusion coefficient for that variable. An analytical solution is given by equation 13 for the variable $\phi$ as a function of the length coordinate $\mathrm{x}$ for $\rho, u$, and $\Gamma$ constant and for prescribed boundary conditions and $x=O$ and $L$. The subscripts $O$ and $L$ represent the bounds of the domain, i.e., the wall thickness, and the parameter $P e$ is the Peclet number, given by equation 14 .

$$
\begin{gathered}
\frac{d}{d x}(\rho u \phi)=\frac{d}{d x} \Gamma \frac{d \phi}{d x} \\
\frac{\phi(x)-\phi_{0}}{\phi_{L}-\phi_{0}}=\frac{\exp \left(P e \frac{x}{L}\right)-1}{\exp (P e)-1}(13) \\
P e=\frac{\rho u L}{\Gamma} \quad(14) \\
T(x)=T_{0}+\left(T_{L}-T_{0}\right)\left(\frac{\exp \left(P e \frac{x}{L}\right)-1}{\exp (P e)-1}\right)
\end{gathered}
$$

Taking $\phi$ to be temperature, equation 13 can be rearranged to provide a simple relationship for the temperature at any location between the two bounds, as given by equation 15. This relationship will provide the basis for the heat recovery model. It is appropriate for application to three-dimensional flows involving complex fluid/solid interactions because the dominant physical processes of heat recovery (convection and diffusion) interact most strongly when they are parallel to one another, i.e., thermal energy conducting through a building envelope is affected most strongly by leaking air flowing parallel to the direction of conduction. We believe this characteristic feature will allow the phenomenon to be modeled effectively using a single directional variable resulting in a one-dimensional model. At this point, $\Gamma$ is left as a generic diffusion coefficient. Later in the model development it will be given a prescribed value, via input, so that it takes on an effective value to represent the composite wall structure.

The next step is to apply equation 15 to a generic building envelope under arbitrary conditions to determine the energy flux through the walls. The external envelope of the 
building used for this analysis is shown in figure 11 along with the relevant variables. This simplified envelope represents the envelope shown in figure 1 with some changes to allow development of the model. The entire building envelope has been separated into two kinds of sections, those that are affected by infiltration and those that are not. Areas of the envelope that are affected by leaking air are represented by A1 and A2 (infiltrating and exfiltrating, respectively) and have both a convection and conduction energy flux. These sections represent parts of the wall in the vicinity of the leaking hole which undergo thermal changes due to infiltration and could potentially represent the entire infiltrating or exfiltrating walls. Areas that are not affected by leaking air are represented by A3 and A4 and have only conduction heat transfer. In the context of figure 1, these sections represent the floor, ceiling, front and back walls, and possibly part of the infiltrating or exfiltrating walls. Since the analysis is one-dimensional, i.e., of a single spatial variable, only two building walls, those on left and right, are needed for the model development. In figure 11, there are no walls on the top, bottom, front, and back because they have been incorporated into the left and right walls. The dashed lines do not represent walls but just show that the left and right walls are connected to form a closed system. The external face of the walls on the left and right form the control volume boundary of the system.

Areas of the envelope that are affected by leaking air are represented in the model as a fluid with the composite properties the fluid/solid/porous wall system. That is, the governing equation used in the model to describe wall sections affected by infiltration, the convectiondiffusion equation, describes a fluid, but is used here to represent the composite wall system. Sections of the wall that are not affected by leaking air are treated as a solid material and are represented with the conduction equation. The six individual flux components shown in figure 11 will be used to determine the infiltration heat recovery factor.

An important implication of the modeling procedure is that details of the system that affect the heat recovery (for example, boundary layers, thermal gradients in the wall, construction details) are lumped and evenly distributed over the entire effective area. The influence of these details is incorporated into the model in an effective manner through proper choice of the effective areas A1 and A2 (described later). Once this is done it is difficult, if not impossible, to make comparisons between the physics of the model and the details of the real system that the model represents. The purpose of the model is to provide select quantitative information about the system, in this case the infiltration heat recovery factor. It cannot provide details about the system, like boundary layer information, which have been lumped together in the modeling process. 


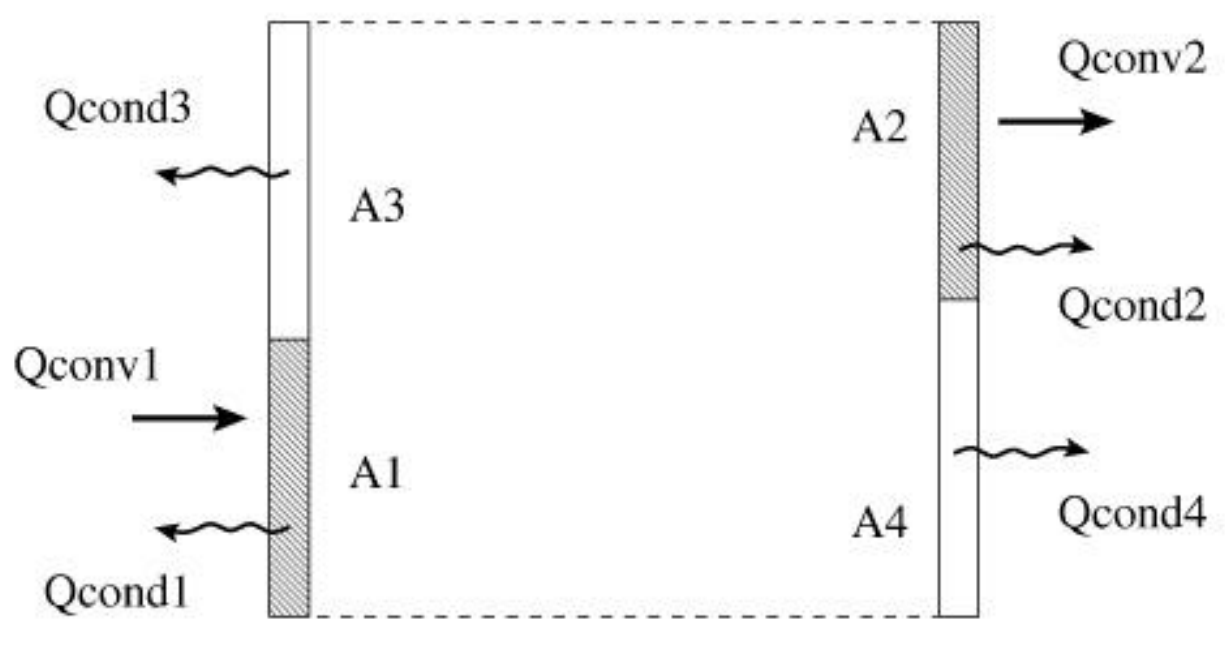

Figure 11: Energy flux through the simplified building envelope used in the model development. Sections A1 and A2 are affected by leaking air and have both convection and conduction terms. Sections A3 and A4 are not affected and have only a conduction term.

The outer faces of the left and right walls form the system control volume boundary.
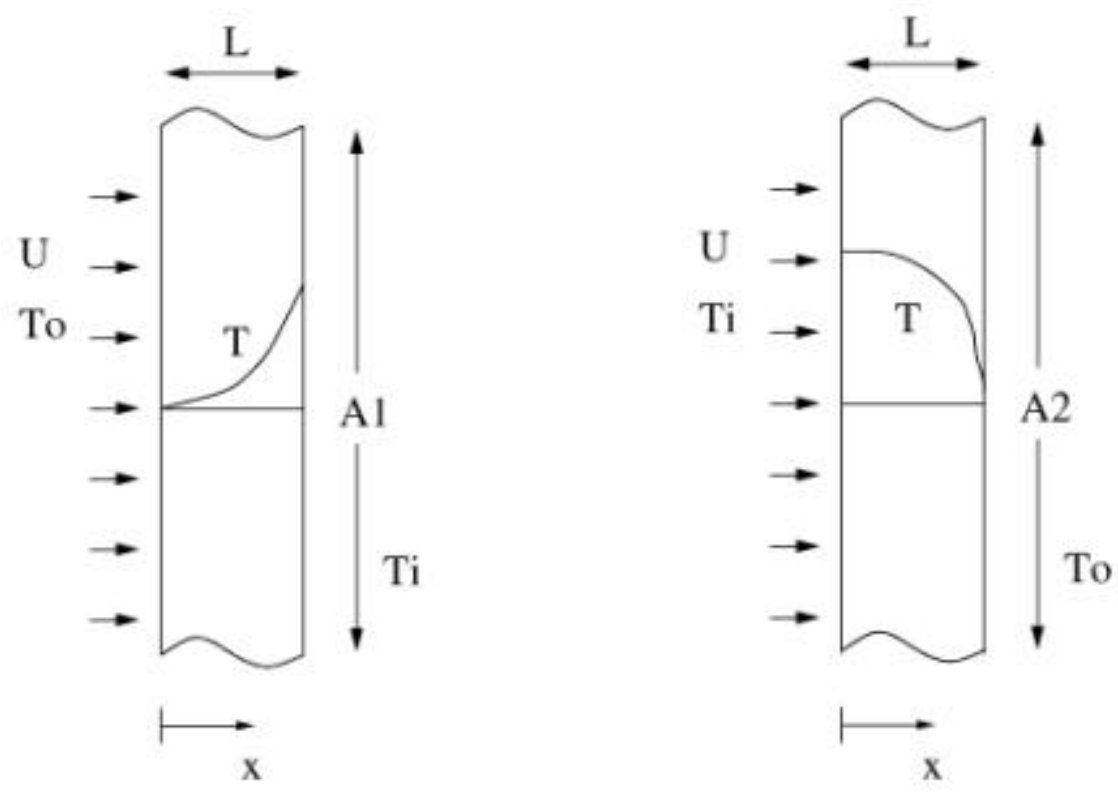

Figure 12: Airflow and thermal profile in the infiltrating (left) and exfiltrating (right) walls, sections A1 and A2, respectively. Air temperatures at the internal and external boundaries are $T_{i}$ and $T_{o}$, respectively. There are no boundary layers shown in the diagram on the inner and outer wall surfaces, but the influence of the boundary layer is incorporated into the model in an effective manner through proper choice of the effective areas A1 and A2. 


\subsection{Convection Terms}

The total energy flux through the building walls has two components, one due to convection $\left(\mathrm{Q}_{\text {conv }}\right)$ and another due to conduction $\left(\mathrm{Q}_{\text {cond }}\right)$. For this initial study, radiation will not be included but may be considered in future work. Each component will be determined across the control volume boundary via equation 15 . There is a convective flux only through the two "effective" areas A1 and A2. These areas do not correspond to the physical area of a hole in the envelope, but to the surface area of the envelope that is affected by infiltrating air. Thus, they are "effective" areas. The actual values for these effective areas, the only parameters not directly input into the model, will be estimated through comparison with CFD data.

$$
\begin{gathered}
Q_{\text {conv }}=Q_{\text {conv } 2}-Q_{c o n v 1} \\
Q_{\text {conv }}=m c_{p} T(x=L)-m c_{p} T(x=0) \\
Q_{\text {conv }}=m c_{p} T_{o}-m c_{p} T_{o} \\
Q_{\text {conv }}=0
\end{gathered}
$$

The net convective energy flux across the effective areas, A1 and A2, is determined in equations 16-19. Equation 16 states that the net convective flux is the flux at the exfiltrating wall minus the flux at the infiltrating wall, as shown in figure 11. Note, in this analysis, energy flow out of the building is considered positive. The air temperatures and mass flow

rates at the control volume boundaries, i.e., the external face of the building envelope, are used in equation 17. The temperatures at the external boundaries, shown in figure 12, are by definition the outside air temperature, as equation 18 reflects. Conceptually, there are no boundary layers at the wall surfaces, but their influence is incorporated into the model in an effective manner through proper choice of the effective areas A1 and A2. Finally, equation 19 gives the interesting result that the net convective flux across the chosen control volume boundary is zero. Therefore, the total effective energy flux due to infiltration in the effective areas will be incorporated into the conduction terms.

\subsection{Conduction Terms}

The total conductive energy flux across the control volume boundary is given by equation 20. It states that the flux is equal to the dot product of the temperature gradient at the control volume boundary (the external wall faces, denoted by the subscript $e$ ) and an outward-pointing normal vector multiplied by the active area and the wall thermal conductivity summed over the entire boundary. Note that the thermal conductivities in the following equations are generic quantities whose values will be determined in an effective manner for the entire wall construction. Equation 21 gives the expanded form of equation 20 when applied to the building envelope shown in figure 11. Note that the normal vectors for envelope areas 1 and 3 point in the negative $\mathrm{x}$-direction and those for areas 2 and 4 point in the positive $\mathrm{x}$-direction resulting in mixed signs for the terms in equation 21. 


$$
\begin{gathered}
Q_{\text {cond }}=\sum_{i=1}^{4}-\left.k_{i} A_{i}(\nabla T \bullet \hat{n})\right|_{e, i} \\
Q_{\text {cond }}=\left.k_{1} A_{1} \frac{d T}{d x}\right|_{e, 1}-\left.k_{2} A_{2} \frac{d T}{d x}\right|_{e, 2}+\left.k_{3} A_{3} \frac{d T}{d x}\right|_{e, 3}-\left.k_{4} A_{4} \frac{d T}{d x}\right|_{e, 4}
\end{gathered}
$$

The gradient terms in equation 21 are evaluated using the solution to the convectiondiffusion equation, given by equation 15 . First, the derivative of temperature is taken with respect to the length coordinate $\mathrm{x}$ and is shown in equation 22. Using the appropriate boundary values, as shown in figure 12 , the gradient terms are evaluated at the infiltrating and exfiltrating walls (A1 and A2) and are given by equations 23 and 24, respectively. This leaves only terms for the inactive areas of the envelope, A3 and A4, to be evaluated. Since these areas are not affected by infiltrating air, their conductive flux and the gradient terms remain constant. The product of the gradient term and thermal conductivity in areas 3 and 4 of the envelope will be represented by a constant as given by equations 25 and 26 , respectively. Finally, the evaluated terms shown in equations 23-26 are placed back into equation 21 to give a new relation for the total conductive flux, equation 27.

$$
\begin{gathered}
\frac{d T}{d x}=\left(T_{L}-T_{0}\right) \frac{P e}{L} \frac{e^{P e \frac{x}{L}}}{e^{P e}-1} \\
\left.\frac{d T}{d x}\right|_{e, 1}=\left(T_{i}-T_{o}\right) \frac{P e_{1}}{L} \frac{1}{e^{P e_{1}}-1} \\
\frac{\left.d T\right|_{e, 2}}{d x}=\left(T_{o}-T_{i}\right) \frac{P e_{2}}{L} \frac{e^{P e_{2}}}{e^{P e_{2}}-1} \\
Q_{\text {cond }}=k_{1} A_{1}\left(T_{i}-T_{o}\right) \frac{P e_{1}}{L} \frac{(24)}{e^{P e_{1}}-1}+k_{2} A_{2}\left(T_{i}-T_{o}\right) \frac{P e_{2}}{L} \frac{e^{P e_{2}}}{e^{P e_{2}}-1}+A_{3} q_{0,3}+A_{4} q_{0,4} \\
q_{0,3}=\left.k_{3} \frac{d T}{d x}\right|_{e, 3} \quad(25) \\
q_{0,4}=-\left.k_{4} \frac{d T}{d x}\right|_{e, 4} \quad(26)
\end{gathered}
$$


The definition for the heat recovery factor is restated in equation 28 with the total energy flux separated into its convective and conductive components. The term $Q_{o}$ represents the total energy flux across the building envelope when there is no infiltration, i.e., pure conduction. For the envelope in this analysis, $Q_{o}$ is represented as the sum of four constant terms as shown in equation 29. Each term in equation 29 accounts for the conductive flux across a particular section in the envelope. Using equations 19, 27, and 29 the numerator in equation 28 can be rewritten as shown in equation 30 .

$$
\begin{gathered}
\varepsilon=1-\frac{\left(Q_{\text {conv }}+Q_{\text {cond }}\right)-Q_{0}}{\dot{m} c_{p}\left(T_{i}-T_{o}\right)} \\
Q_{0}=A_{1} q_{0,1}+A_{2} q_{0,2}+A_{3} q_{0,3}+A_{4} q_{0,4} \\
Q_{\text {conv }}+Q_{\text {cond }}-Q_{0}=k_{1} A_{1}\left(T_{i}-T_{o}\right) \frac{P e_{1}}{L} \frac{1}{e^{P e_{1}}-1}+k_{2} A_{2}\left(T_{i}-T_{o}\right) \frac{P e_{2}}{L} \frac{e^{P e_{2}}}{e^{P e_{2}}-1}-A_{1} q_{0,1}-A_{2} q_{0,2}
\end{gathered}
$$

At this point, the constant terms used to account for conduction through the envelope will be examined. In reality, heat conduction through a building wall is dependant on a host of factors including construction details of the wall, thermal properties of the wall materials, environmental conditions, and air flow in the vicinity of the wall. However, these details cannot be incorporated into this one-dimensional model, so a simplified approach is taken following conventional practice in building energy simulation. It is assumed that there is a linear temperature profile through the wall and the heat flux obeys Fourier's law for heat conduction(13), equation 20. With these assumptions, the conduction heat flux through the wall with no infiltration, $q_{o}$, is expressed in equations 31 and 32.

$$
\begin{gathered}
q_{0,1}=\left.k_{1} \frac{d T}{d x}\right|_{e, 1}=k_{1} \frac{T_{i}-T_{o}}{L} \\
q_{0,2}=-\left.k_{2} \frac{d T}{d x}\right|_{e, 2}=-k_{2} \frac{T_{o}-T_{i}}{L}
\end{gathered}
$$

The information from equations $30-32$ can be inserted into equation 28 to provide a new relationship for the heat recovery factor, given by equation 33 . Note, this expression shows that the heat recovery for the entire building envelope is dependent only on what happens in the active areas. Of course, a fundamental assumption of this model is that the envelope can be divided into separate, non-interacting regions, some being affected by infiltration and some not. The heat recovery factor is a function of the wall thermal 
conductivity, active area, wall thickness, and Peclet number for both the infiltrating and exfiltrating walls.

$$
\boldsymbol{\varepsilon}=1-\frac{k_{1} A_{1} \frac{P e_{1}}{e^{P e_{1}}-1}+k_{2} A_{2} \frac{P e_{2} e^{P e_{2}}}{e^{P e_{2}}-1}-\left(k_{1} A_{1}+k_{2} A_{2}\right)}{m c_{p} L}
$$

\subsection{Introduction of a Dimensionless Flow Rate}

The final step is to recast the Peclet number in terms of quantities that are meaningful for this application. This is an important part of the modeling process, which can be considered a modeling step in itself. Equation 34 shows the definition of the Peclet number. The velocity, $u$, in equation 34 is replaced by the relation shown in equation 35 where $m$ is the mass flow rate of leaking air, $\rho$ is the air density, and $A$ is the active area. Next, the thermal conductivity, $k$, is replaced by the U-value for the wall via equation 36 , which makes use of the relation for conduction given by equation 31 . This step replaces the micro-scale material property, $k$, with the macro-scale effective U-value for the composite wall system. The Peclet number is transformed into a dimensionless flow rate, $a$, defined by Claridge and Bhattacharyya (8), using effective values characteristic to the system as shown in equation 37.

$$
\begin{aligned}
& P e=\frac{\rho u L}{k / c_{p}} \\
& u_{i}=\frac{m}{\rho A_{i}} \\
& U_{i}=\frac{q_{o, i}}{\Delta T}=\frac{k_{i}}{L} \\
& a_{i}=\frac{m c_{p}}{U_{i} A_{i}}
\end{aligned}
$$

Using the relations in equations 33-37, a new expression is created for the heat recovery factor and is given by equation 38. Equation 38 shows that the heat recovery factor is a function only of the dimensionless flow rate, $a_{i}$, for the infiltrating and exfiltrating walls. When presented in this form the symmetry between infiltration and exfiltration is apparent. It is assumed that in practice the leakage rate and the U-value can be measured or estimated. 
That leaves only the effective area to be determined before the heat recovery factor can be calculated. This will be done here through comparison with CFD simulation results.

$$
\varepsilon=-\frac{1}{e^{a_{1}}-1}-\frac{1}{e^{a_{2}}-1}+\frac{1}{a_{1}}+\frac{1}{a_{2}}
$$

\subsection{Area Ratios in the Heat Recovery Factor Equation}

One last change will be made in the expression for the heat recovery factor, a simplification for ease of use. A value is determined for the non-dimensional flow rate for the entire structure, denoted as $a_{o}$, using the overall U-value and the total surface area of the building, denoted as $A$, as shown in equation 39. This is convenient because the $U A$ value for a building is a familiar quantity that can be measured or estimated. Both $a_{1}$ and $a_{2}$ can now be expressed in terms of $a_{o}$ and the unknown effective areas can be extracted into separate parameters as area ratios weighted by the U-values, as equations 40 and 41 show. The final form of the expression for the heat recovery factor is given by equation 42 . It shows that the heat recovery factor for a given building depends on the UA of the building and the infiltration rate, which appear as $a_{o}$, and the effective areas for infiltration and exfiltration, which appear as the effective area ratios, $f_{1}$ and $f_{2}$.

$$
\begin{gathered}
a_{o}=\frac{m c_{p}}{U A} \\
f_{1}=\frac{a_{o}}{a_{1}}=\frac{U_{1} A_{1}}{U A} \\
f_{2}=\frac{a_{o}}{a_{2}}=\frac{U_{2} A_{2}}{U A} \\
\varepsilon=-\frac{1}{e^{a_{o} / f_{1}}-1}-\frac{1}{e^{a_{o} / f_{2}}-1}+\frac{f_{1}+f_{2}}{a_{o}}
\end{gathered}
$$

\subsection{Plots of the Heat Recovery Factor}

Some plots of the heat recovery factor, calculated with equation 42, are shown in figures 13 and 14. These plots show the range of potential values for the heat recovery factor. The curves shown in figure 13 each have different values for the area ratios, as shown in the legend, with the individual area ratios $\left(f_{l}\right.$ and $\left.f_{2}\right)$ being equal for a given curve. Note, the maximum theoretical value possible for the sum of the area ratios is1.0, which 
corresponds to 100 percent of the building envelope being active in the infiltration process. The curve with each ratio equal to 0.5 corresponds to this situation. Figure 13 shows that the heat recovery factor is high at small infiltration rates and drops as the infiltration rate increases. The transit time of air leaking through the wall decreases as the flow rate increases, allowing less time for heat transfer to occur between the leaking air and wall structure. This reduces heat recovery. Also, the plot shows that the heat recovery drops with decreasing area ratios. The ratio corresponds to the fraction of the wall that interacts with leaking air. When only a small fraction of the envelope interacts with leaking air, its impact is small and the resulting heat recovery is low, as the case with $f=0.025$ shows. When the ratio is large, the heat recovery is higher.

Figure 14 shows the heat recovery factor curves for four cases. Two of the cases have equal values for the individual area ratios, $f_{1}$ and $f_{2}$, and are taken from figure 13 . The other two have unequal values for the individual ratios, but the sum of the ratios is equal to that of the corresponding curve with equal area ratios. The top two curves in figure 14 have a total effective area of 100 percent, and the bottom two curves have a total effective area of 20 percent. Actual values used for the ratios are shown in the legend. The same overall behavior in the heat recovery factor is seen in all the curves. However, at low infiltration rates, there are small differences between curves that have the same total value for the area ratios but different individual values. In both comparisons, the cases with unequal ratios have a lower heat recovery than the corresponding case with equal ratios. These results indicate that for a given leakage rate heat recovery is highest when the effective area is large and the individual areas (infiltrating and exfiltrating) are equal.

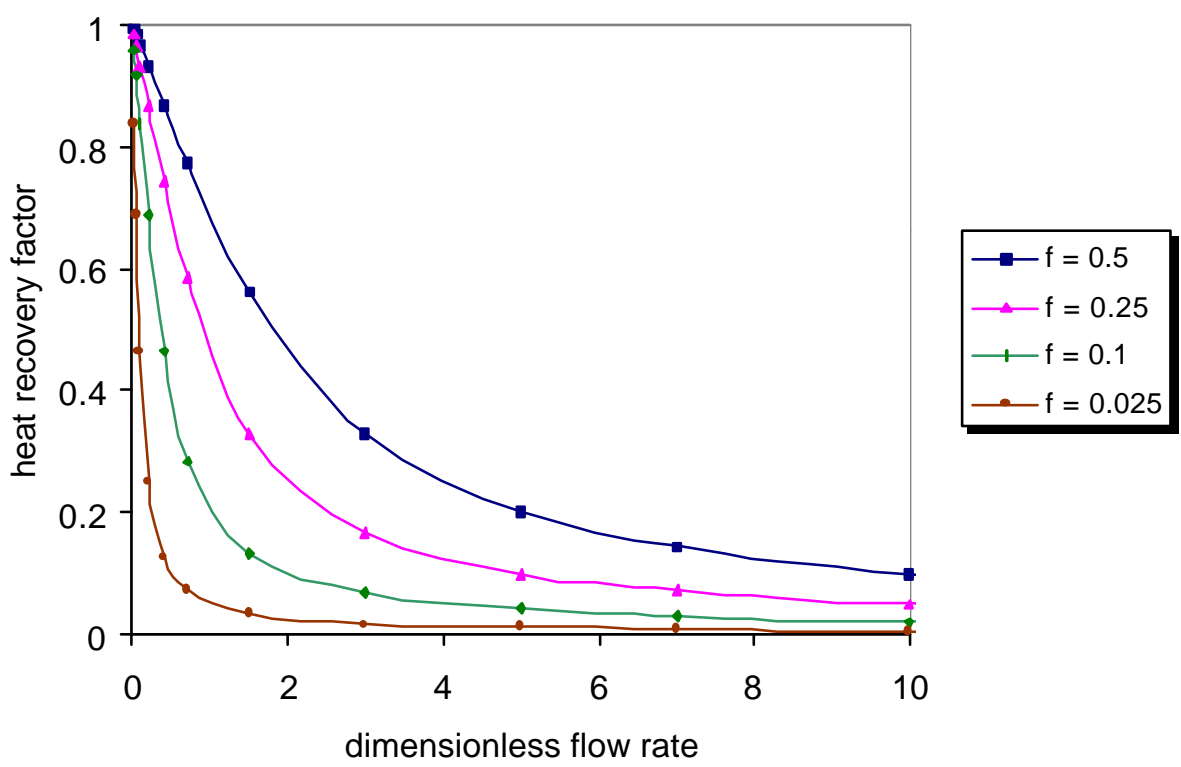

Figure 13: Heat recovery factor calculated with the simplified model using equal area ratios $\left(f_{1}=f_{2}=f\right)$. Note that the heat recovery drops with decreasing area ratios and increasing leakage rate. 


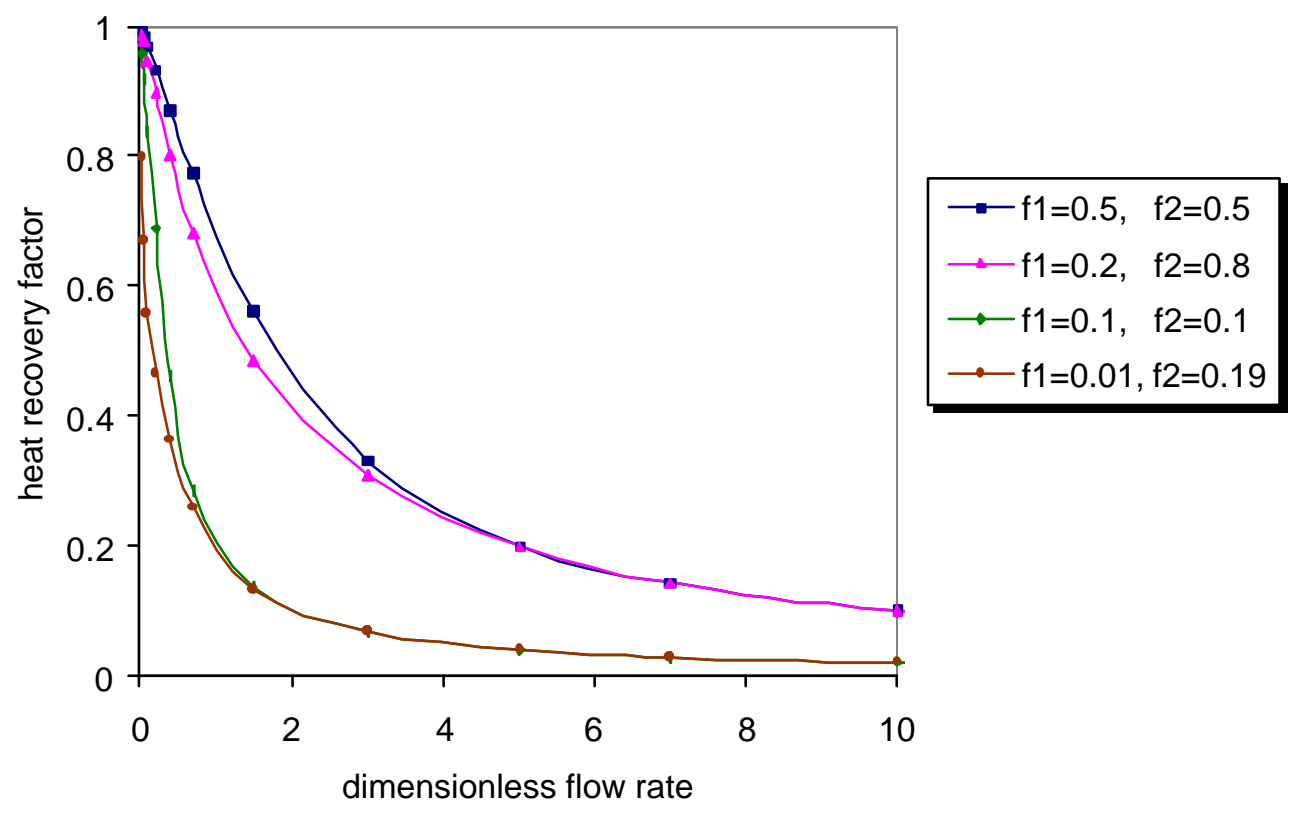

Figure 14: Heat recovery factor calculated with the simplified model using unequal area ratios. For a given sum of area ratios $\left(f_{1}+f_{2}\right)$ the heat recovery is highest when the individual ratios are equal.

\subsection{Comparison of Mathematical Model with CFD Results}

Predictions of the heat recovery factor made with the mathematical model, equation 42 , are now compared to values determined from CFD simulations. In these comparisons, the area ratios in the model, $f_{1}$ and $f_{2}$, are adjusted to provide the best overall agreement as determined by a least-squares fit. The ratios are given equal values here because the infiltrating and exfiltrating walls used in the CFD simulations were symmetric.

Figure 15 shows heat recovery values for the walls with a high/low hole configuration determined from 2D and 3D CFD simulations and the model. The value for the area ratio was adjusted to give the best agreement, which, for this case, was found to be 0.33 , or 33 percent of the wall area. The model predictions show fairly good agreement with CFD results, but do not match exactly. Better agreement would occur if the model predictions were slightly lower at small leakage rates and slightly higher at large leakage rates. 


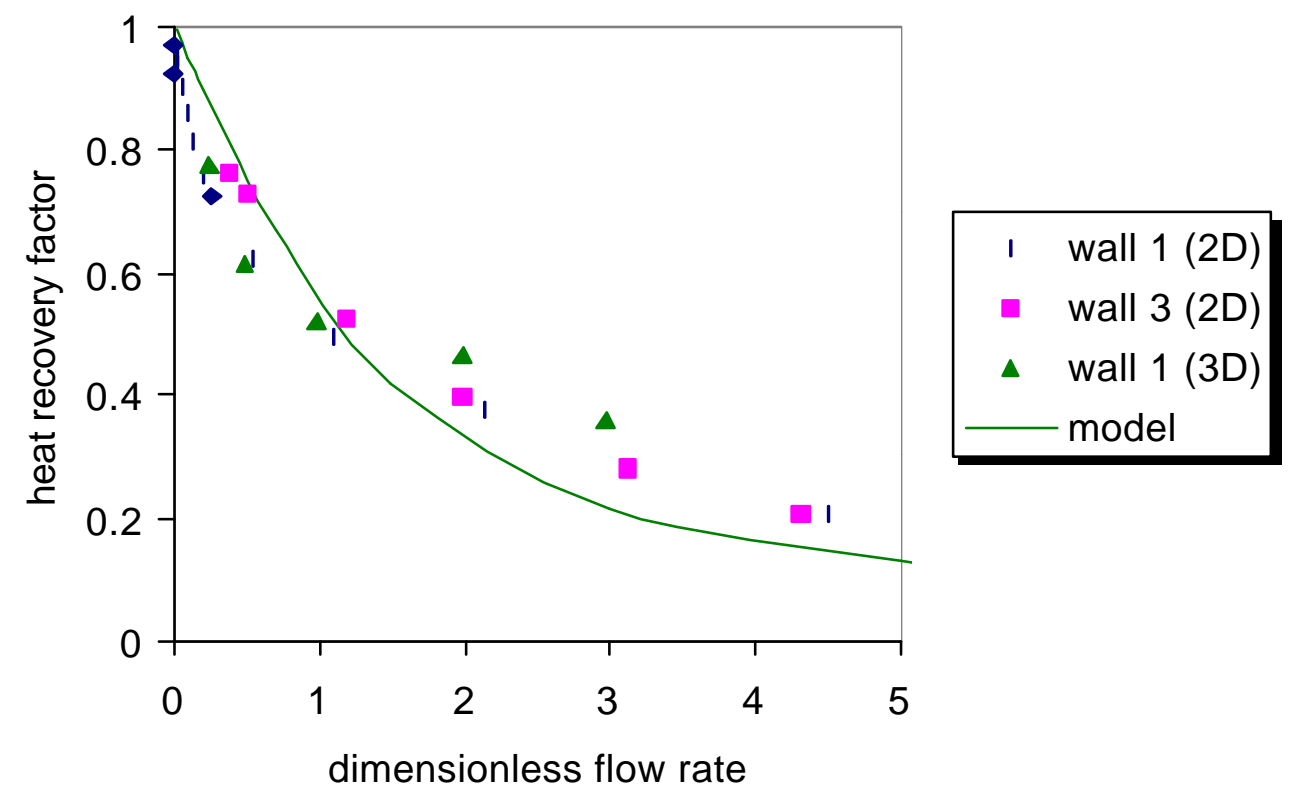

Figure 15: Heat recovery factor for walls with a high/low hole configuration (insulated and empty wall cavities) determined from CFD simulations and the simplified model (equation 42) using a constant area ratio $(f=0.33)$.

Figure 16 shows heat recovery values for the walls with a straight through hole configuration determined from 2D and 3D CFD simulations and the model. For this case, the best agreement was achieved with a value of 0.18 for the area ratio, or 18 percent of the wall area. Agreement is good, but, as before, agreement would be better if the model predictions were slightly lower at small leakage rates and slightly higher at large leakage rates. 


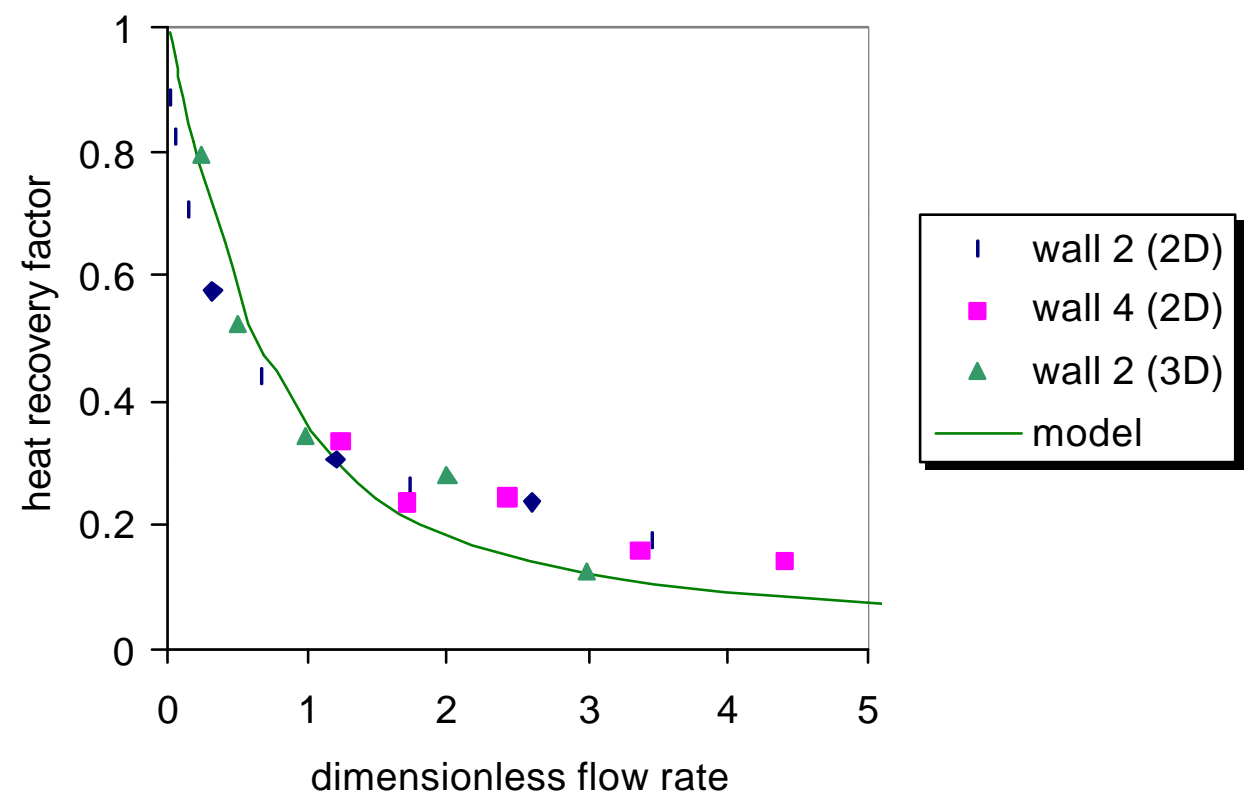

Figure 16: Heat recovery factor for walls with a straight through hole configuration (insulated and empty wall cavities) determined from CFD simulations and the analytical model (equation 42) using a constant area ratio $(f=0.18)$.

The results in figures 15 and 16 suggest that our simple model does not capture the full physics of the problem. While the trend is generally correct, the model predictions decrease faster at high flow rates than the CFD data does. Some of the CFD results suggest that part of the heat recovery occurs in the thermal boundary layers on each side of the wall and part occurs within the wall. For example, cool infiltrating air falls along the inner surface of the wall reducing the conductive heat loss. Also, some of the air sucked into the leak from the external boundary layer will be at a higher temperature (a smaller overall temperature difference) and will mitigate the infiltration load. This effect can be seen by looking at the air flow velocity vectors from one of the two-dimensional CFD simulations shown in figure 17 . This effect would imply that even with no heat exchange within the wall itself there would be some heat recovery. We expect this effect (sucking in the thermal boundary layer) to be the dominant mechanism for heat recovery at higher leakage rates. Additionally, non-uniform air flows within the wall could enhance this effect (e.g., through interaction with convective cells).

We believe these effects contribute to heat recovery, but they are not explicitly included in our model. Therefore, we consider the possibility of a variable area ratio in which the area is augmented to include the effects of the boundary layer and other convective interactions. The variable area ratio model must meet certain physical constraints. When there is very little air leakage, the area ratio and the boundary layer effect should be small. Also, there should be a positive definite increase in the magnitude of the area ratio with increasing leakage to an asymptote that must never be greater than one. 


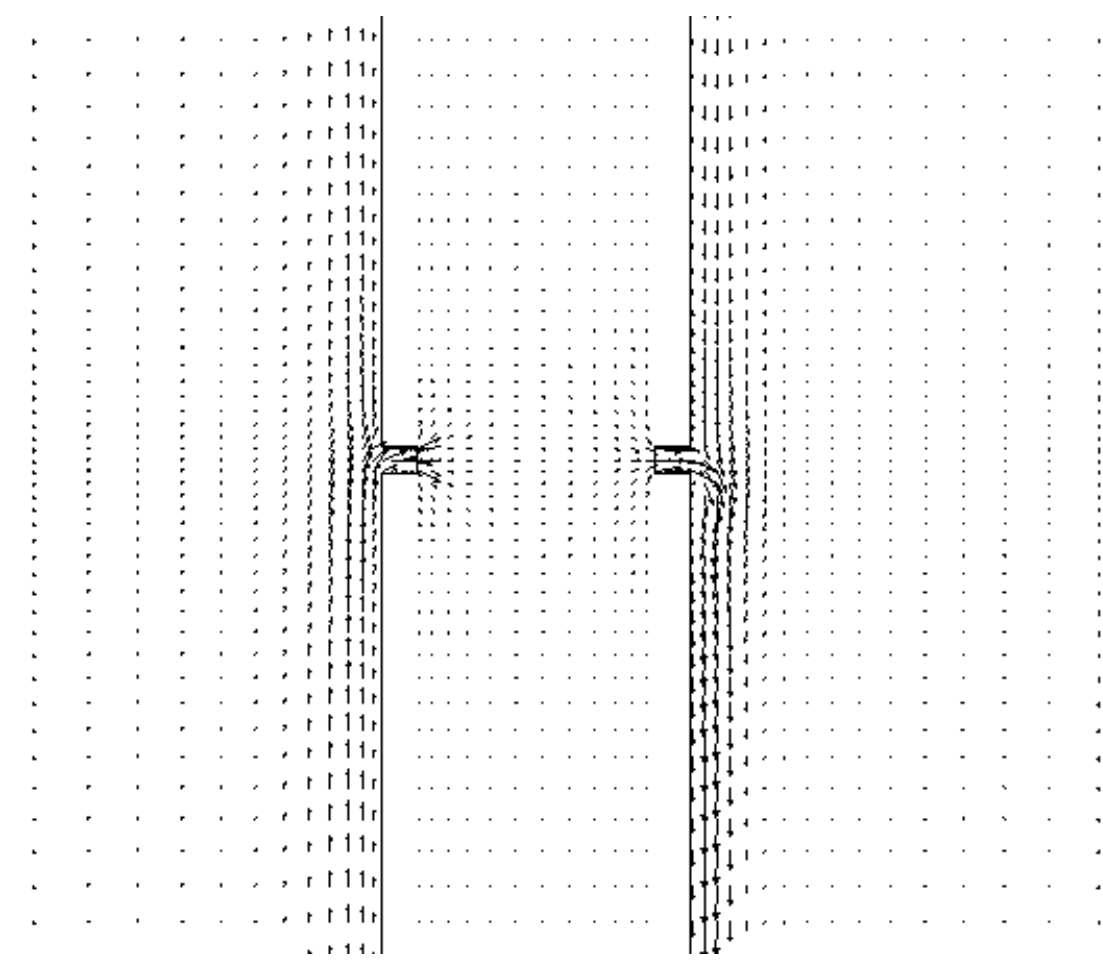

Figure 17. Air flow velocity vectors from a two-dimensional CFD simulation of the wall with a straight through leakage path (wall 2). It shows how the external boundary layer can be sucked into the leak and infiltrating air can be held near the interior wall surface.

To test the efficacy of a variable area ratio representation calculations are performed to determine the ratio magnitude needed to make the analytical model predictions of the heat recovery factor agree exactly with those determined from CFD. This is done at every CFD data point for empty and insulated walls with both high/low and straight through leakage configurations. Results for the high/low configuration are shown in figure 18 and results for the straight through configuration are shown in figure 19. In both cases, the area ratio starts off small, close to zero, at low leakage rates, as expected. As the leakage rate increases, the area ratio increases and appears to level off at higher leakage rates. The area ratio for the high/low configuration increases faster than that for the straight through and levels off at a larger value. Again, this is the expected behavior based on physical constraints. There are some outlying points in figures 18 and 19, which correspond to the 3D data. We are not certain why these points are outlying from the general trend, but it could be due to numerical error in the calculations related to insufficient grid resolution. This analysis suggests that a variable representation for the area ratio based on leakage rate could increase the accuracy of the analytical model. The exact mathematical form for this relation and the physical basis for the form are now under investigation. 


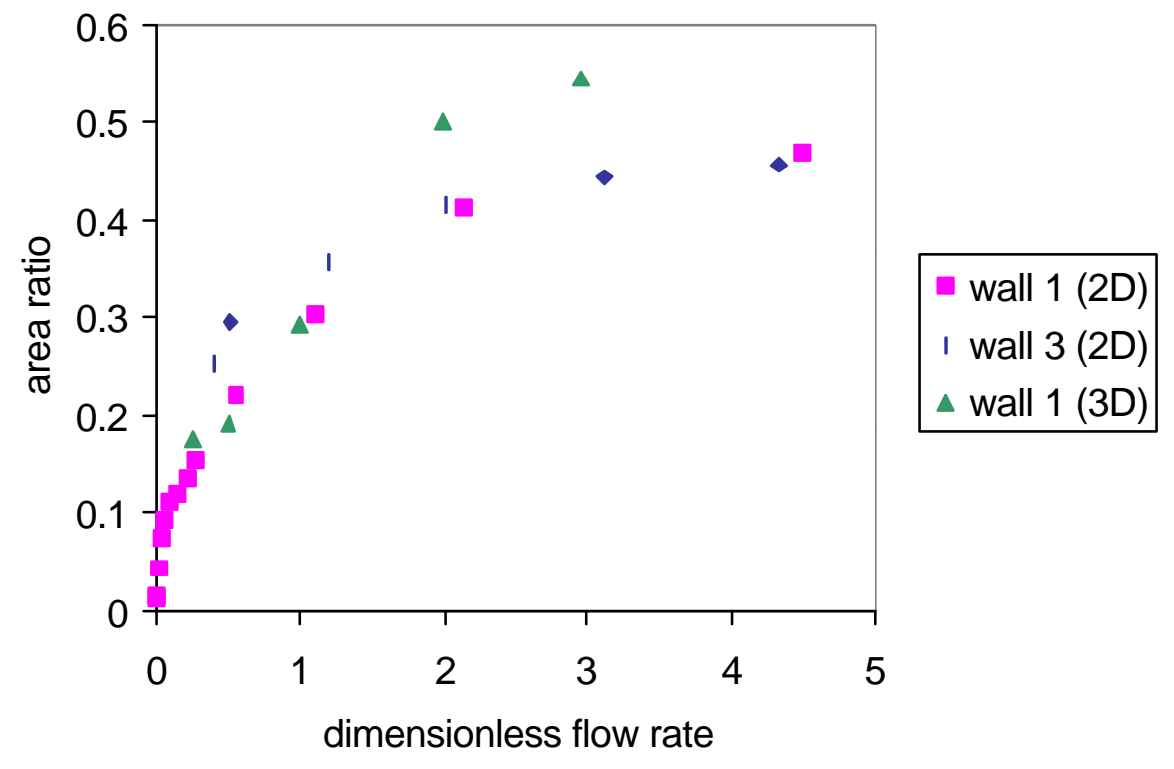

Figure 18: Area ratio for walls with a high/low hole configuration (insulated and empty wall cavities) determined by matching the analytical model (equation 42) predictions to CFD heat recovery factors at individual CFD data points.

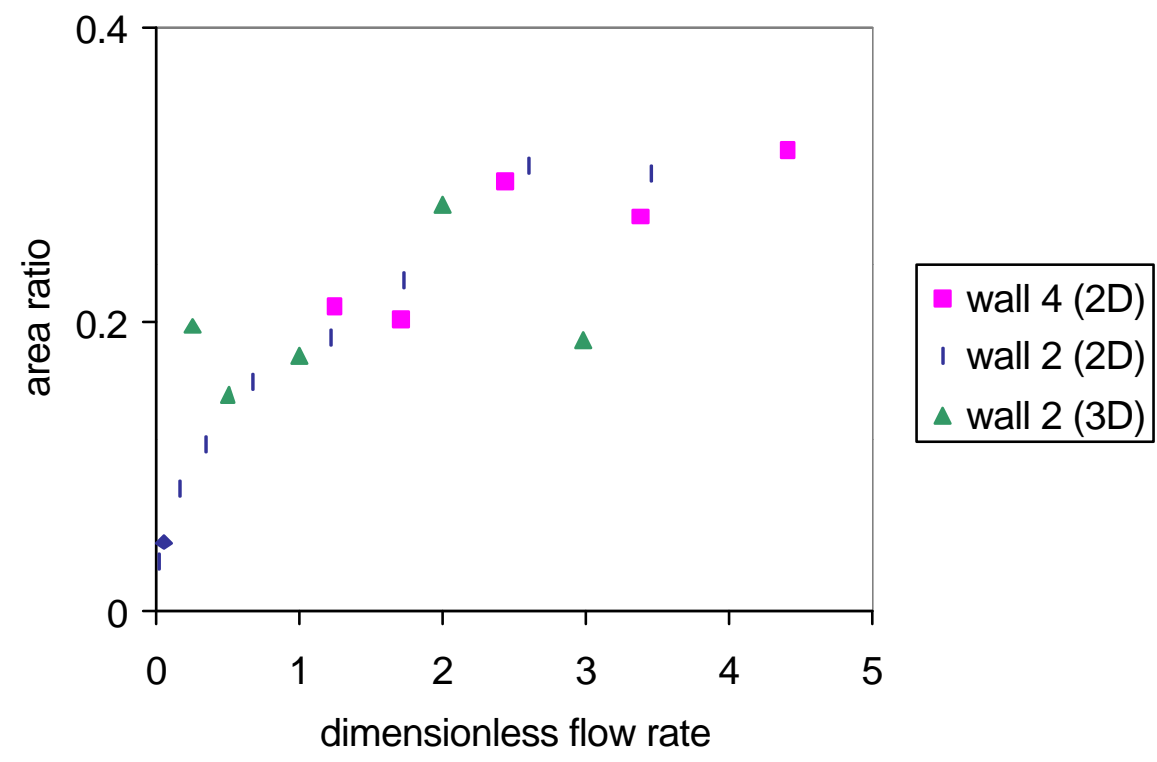

Figure 19: Area ratio for walls with a straight through hole configuration (insulated and empty wall cavities) determined by matching the analytical model (equation 42) predictions to CFD heat recovery factor at individual CFD data points. 


\section{Conclusions}

In this study, CFD simulations were used to examine the fundamental physics of the infiltration heat recovery process. Results were found to compare well (within about 10 percent) with limited published laboratory data corresponding to one of the leakage scenarios examined. Also, a simple, robust macro-scale mathematical model was developed that can accurately predict the heat recovery factor for air infiltrating through building walls when supplied with the proper input. The required inputs are the building U-value, the leakage rate, and an effective area for infiltration and exfiltration. The effective areas were determined here for specific leakage geometries through comparison with CFD data. The leakage geometries examined may be fundamental cases that can be combined to create most other leakage scenarios. Over the wide range of airflows considered, model predictions agree fairly well with CFD data. However, it appears that better agreement can be attained by using a variable relation, based on leakage rate, for the effective area. The exact mathematical form for this relation and the physical basis for the form are now under investigation.

These results show the potential importance of infiltration heat recovery. The extent of heat recovery was found to be dependent on the leakage path geometry, infiltration flow rate, and wall construction. In some cases with low infiltration rates and long leakage paths, the heat recovery can be substantial, well over 80 percent. In these cases, the classical method would over-predict the extra heating load due to infiltration (see appendix 4 for a detailed example). According to these findings, under typical leakage conditions for most residential buildings $\left(a_{o} \leq 1\right)$ the heat recovery could be around 40 percent. Even at infiltration rates that would be considered very high for residential buildings $\left(a_{o}=5\right)$ the heat recovery is still sizeable at about 20 percent. In this situation, the conventional prediction for the infiltration load would be in error by 20 percent.

All possibilities have not been examined in this study, but it is clear that some modification could be made to the conventional method for prediction of infiltration energy loads to increase its accuracy. The model for infiltration heat recovery presented in this paper could easily be incorporated into whole-building energy analysis programs to provide improved predictions for the energy impact of steady-state infiltration. An important implication of the modeling procedure is that details of the system that affect heat recovery (for example, boundary layers, thermal gradients in the wall, construction details) are lumped and evenly distributed over the entire effective area. The influence of these details is incorporated into the model in an effective manner through proper choice of the effective areas. Once this is done it is difficult, if not impossible, to make detailed comparisons between the physics of the model and the reality that the model represents. The purpose of the model is to provide select quantitative macro-scale information about the system, in this case the infiltration heat recovery factor. It cannot provide detailed information about the system, like boundary layer structure, which have been lumped together in the modeling process. 


\section{References}

1) Baker, P. H., Sharples, S., Ward, I. C. (1987) “Air flow through cracks”, Building and Environment, Vol. 22, No. 4, pp. 293-304.

2) Bhattacharyya, S., Claridge, D. E. (1995) "The energy impact of air leakage through insulated walls", Transactions of the ASME, Vol. 112, pp. 132-139.

3) Brunsell, J. T. (1995) "The indoor air quality and the ventilation performance of four occupied residential buildings with dynamic insulation", $16^{\text {th }}$ AIVC Conference:

Implementing the results of ventilation research, Palm Springs, USA, September 18-22, Proceedings Vol. 2, pp. 471-482.

4) Buchanan, C.R., Sherman, M. H. (1998) "Simulation of Infiltration Heat Recovery", $19^{\text {th }}$ AIVC Annual Conference, Oslo, Norway, September, 1998.

5) Burns, P. J., Chow, L. C., Tien, C. L. (1977) "Convection in a vertical slot filled with porous insulation”, Int. J. Heat Mass Transfer, Vol. 20, pp. 919-926.

6) Caffey, G. E. (1979) “Residential air infiltration”, ASHRAE Trans., Vol. 85, pp. 41-57.

7) Claridge, D. E., Liu, M. (1996) "The measured energy impact of infiltration in an outdoor test cell”, Transactions of the ASME, Vol. 118, pp. 162-167.

8) Claridge, D. E., Bhattacharyya , S. (1990) "The measured impact of infiltration in a test cell”, J. Solar Energy Engineering, Vol. 117, pp. 167-172.

9) Etheridge, D. W. (1988) "Modelling of air infiltration in single- and multi-cell buildings", Energy and Buildings, Vol. 10, pp. 185-192.

10) Jensen, L. (1993) "Energy impact of ventilation and dynamic insulation", $14^{\text {th }}$ AIVC Conference: Energy impact of ventilation and air infiltration, Copenhagen, September 21-23, Proceedings, pp. 251-260.

11) Kohonen, R., Virtanen, M. (1987) "Thermal coupling of leakage flow and heating load of buildings", ASHRAE Trans., Vol. 93, pp. 2303-2318.

12) Liddament, M. W. (1987) “Power law rules-- OK?”, Air Infiltration Review, Vol. 8, No. 2, pp. 4-6.

13) Mills, A. F. (1992) Heat Transfer. Irwin, Burr Ridge, Illinois

14) "NIST estimates nationwide energy impact of air leakage in U. S. buildings" (1996) J. Research of NIST, Vol. 101, No. 3, p. 413.

15) Persily, A. (1982) "Understanding air infiltration in homes", Report PU/CEES No. 129, Princeton University Center for Energy and Environmental Studies, February, p. 335.

16) Sherman, M., Matson, N. (1993) "Ventilation-Energy liabilities in U.S. dwellings", $14^{\text {th }}$ AVIC Conference: Energy impact of ventilation and air infiltration, Copenhagen, September 21-23, Proceedings, pp. 23-41. 
17) Versteeg, H. K., Malalasakera, W. (1995) Introduction to Computational Fluid Dynamics. Longman Scientific \& Technical, New York.

18) Virtanen, M., Heimonen, I., Kohonen, R. (1992) "Application of the transfer function approach in the thermal analysis of dynamic wall structures", ASHRAE/DOE/BTECC Conference: Performance of the Exterior Envelopes of Buildings, December 7-10, Clearwater Beach, Florida, USA, Proceedings.

\section{Appendix 1: Infiltration Basics}

\section{App.1.1: Energy flux through the building envelope}

In this analysis two modes of energy transfer through the building envelope are considered: conduction, mainly through the walls, and convection, via infiltrating air. These are shown graphically in Figure 1.App. In reality, energy transport also occurs through radiation, but we think infiltration has little impact on this load and vice versa. This hypothesis will be tested in future work. Also, convective transport occurs via the HVAC system intake and exhaust, but this has no direct effect on infiltration heat recovery so it will not be considered here. The infiltration load and conduction load interact strongly and, therefore, are the most important quantities to study in order to understand the infiltration heat recovery phenomenon.

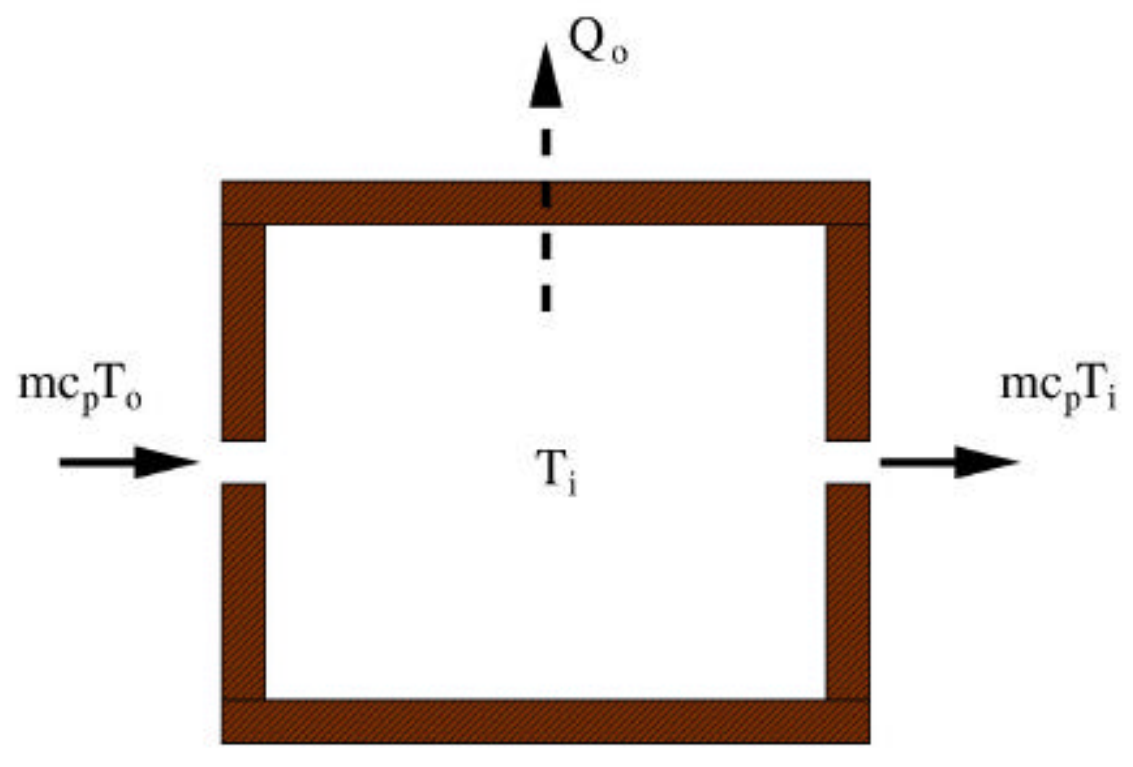

Figure 1.App: Cross section of a generic building envelope showing the conventional conduction and infiltration energy load terms. $Q_{o}$ is the conduction energy load with no

infiltration and $m c_{p}\left(T_{i}-T_{o}\right)$ is the infiltration energy load, where $T_{i}$ is the indoor air temperature, $T_{o}$ is the outdoor air temperature, and $\mathrm{m}$ is the leakage rate. Air leaking into the building is said to be infiltrating and air leaking out of the building exfiltrating. 


\section{App.1.2: Conventional method of accounting for infiltration energy load}

In the absence of infiltration through the building envelope, the energy load on the building, not considering radiation and HVAC intake/exhaust, is that purely from conduction, denoted as $Q_{o}$. When there is infiltration the conventional method of accounting for the extra energy load, $Q_{\text {infC }}$, is to add a simple convective term to the energy balance for the building that is based on the leakage rate and the indoor and outdoor air temperatures. The conventional relationship used to calculate the total energy load with infiltration, denoted as $Q_{C}$, is based on the system shown in figure 1.App and is given by equation 1.App. This relationship assumes there is no interaction (heat transfer, moisture deposition, etc.) between the leaking air and building walls.

$$
Q_{C}=Q_{o}+Q_{\mathrm{inf} C}=Q_{o}+m c_{p}\left(T_{i}-T_{o}\right)
$$

\section{App.1.3: Including Infiltration Heat Recovery in the conventional method}

In reality, there is heat transfer between the building walls and leaking air as is enters (infiltration) and exits (exfiltration) the building envelope. The result is that the actual values for the conduction, infiltration, and total loads are different from that predicted by equation 1.App. This case is shown graphically in figure 2.App. with the appropriate variables. The relationship for the actual total energy load is given by equation 2.App., in which $Q_{o}{ }^{*}, Q_{\text {inf }}$, and $Q$ are the actual conduction, infiltration, and total loads, respectively.

$$
Q=Q_{o}{ }^{*}+Q_{\mathrm{inf}}=Q_{o}{ }^{*}+m c_{p}\left(T_{2}-T_{1}\right)
$$

In the case of a warm inside and cool outside $\left(T_{i}>T_{o}\right)$, heat transfer within and near the surface of the building envelope walls would cause infiltrating air to enter the building at a temperature greater than that outside and exfiltrating air to exit at a temperature less than that inside. The actual temperatures of infiltrating and exfiltrating air $\left(T_{1}\right.$ and $T_{2}$, respectively, taken at some consistent system boundary) are not known and are dependent on a host of factors, but this implies that, in general, the infiltration energy load is less than that predicted by the conventional method. The effect on the conduction load is not known, however. Since the process is asymmetric in this study (conduction decreased by infiltration and increased by exfiltration), it is thought that the net effect on the conduction load is small. This suggests that the actual total energy load is less than that predicted by the conventional method, i.e., $Q<Q_{C}$. In reality, the infiltrating and exfiltrating leakage paths will most likely not be completely symmetric and the actual effect on the conduction load will depend on the leakage characteristics of the specific structure. This topic will be explored in future work.

$$
Q=Q_{o}+(1-\varepsilon) m c_{p}\left(T_{i}-T_{o}\right)
$$

In light of the situation, i.e., an unknown actual conduction load thought to be close to the conduction load with no infiltration and a reduced infiltration load with unknown actual 
air temperatures, an obvious and simple method of accounting for the effect of infiltration heat recovery is to insert a reduction factor into the conventional relationship for the total building energy load, equation 1.App., so that it provides the actual total energy load. The reduction factor is multiplied by the infiltration term, since this term is known to be reduced by heat recovery, and the conduction term is represented by the conduction load with no infiltration. Equation 3.App utilizes this accounting method to give the actual total energy load. The quantity $\varepsilon$ in equation 3.App is called the infiltration heat recovery factor and accounts for the net effect of heat transfer between leaking and building walls. Note, the values of the individual terms in equation 3.App probably do not accurately predict the actual values but the sum total is correct if $\varepsilon$ is properly chosen. This is a convenient and useful method because the parameters in equation 3.App $\left(Q_{o}, m, T_{i}, T_{o}\right)$ should all be known. This reduces the problem to simply determining $\varepsilon$, as compared to determining the actual values of the individual terms, which could be very difficult.

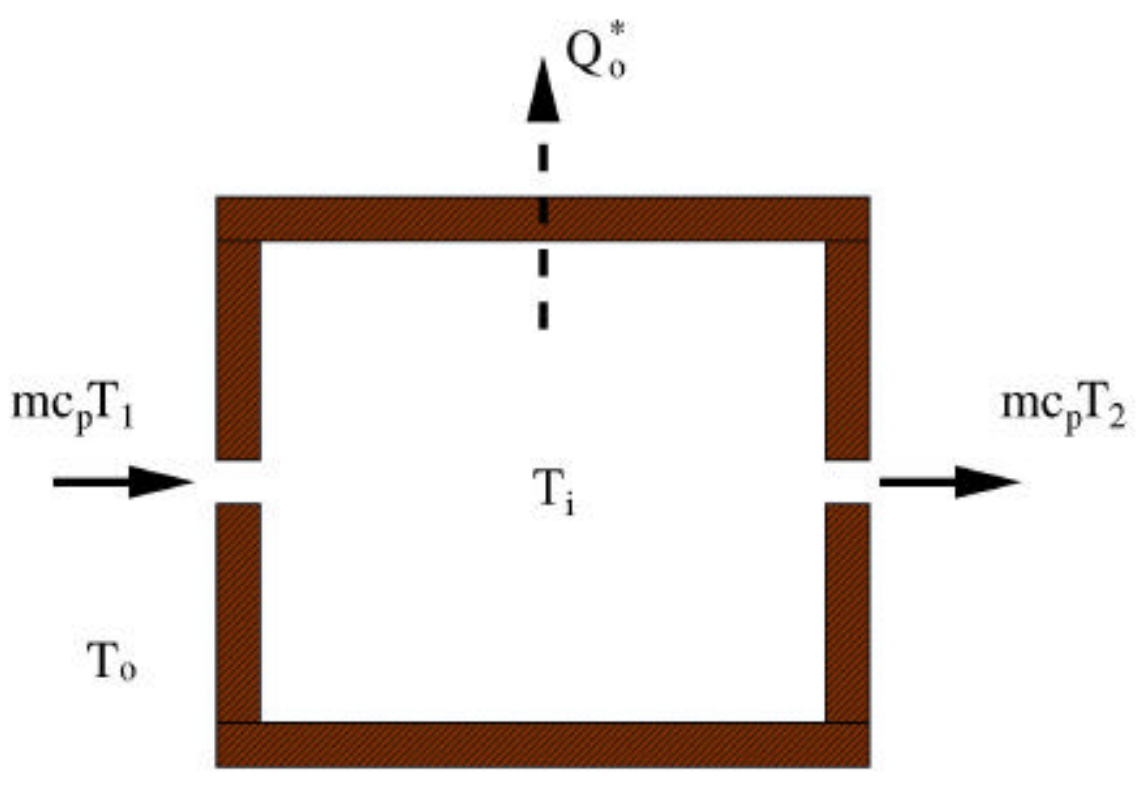

Figure 2.App: Cross section of a generic building envelope showing the actual conduction and infiltration energy load terms. $Q^{*}{ }_{o}$ is the actual conduction energy load with infiltration and $m c_{p}\left(T_{2}-T_{1}\right)$ is the actual infiltration energy load, where $T_{2}$ is the exfiltrating air temperature, $T_{1}$ is the infiltrating air temperature (taken at the system control volume boundary), and $\mathrm{m}$ is the leakage rate. Note that for $T_{i}>T_{o}$, in general $T_{1}>T_{o}$ due to warming and $T_{2}<T_{i}$ due to cooling and the relation between $Q^{*}{ }_{o}$ and $Q_{o}$ is unknown but they are thought to be nearly equal in size.

\section{Appendix 2: CFD Details}

\section{App. 2.1: Wall modeling and boundary conditions}

In the CFD simulations, several mathematical equations are used within the computational domain to represent different components of the leaking building wall system 
under investigation. A schematic showing a cross-section of the system is given in figure 3.App. for a single wall (infiltrating or exfiltrating) with an insulated wall cavity.

This schematic illustrates how various equations and boundary conditions are combined spatially to create the leaking building wall system. Note, results from two or more walls are added together to provide information for a complete room system.

The outer border of the system supplies boundary conditions (temperature, pressure, velocity) for the simulation. As figure 3.App shows, the left and right sides of the system boundary are composed of a wall with zero flow velocity and fixed thermal boundary conditions and an inlet or outlet with fixed thermal and pressure (or velocity) boundary conditions. The region within the boundary is comprised entirely of live cells (control volumes). Calculations are performed for each live cell using the appropriate governing equations to determine numerical values for the relevant variables in that cell. In cells that represent air, the Navier-Stokes and energy equations (eq. 4-6) are the governing equations. Cells that represent the wall sheathing are governed by the conduction equation (eq. 7). Cells that represent insulation are governed by Darcy's Law and a modified energy equation (eq. 89). This information is shown graphically in figure 3.App.

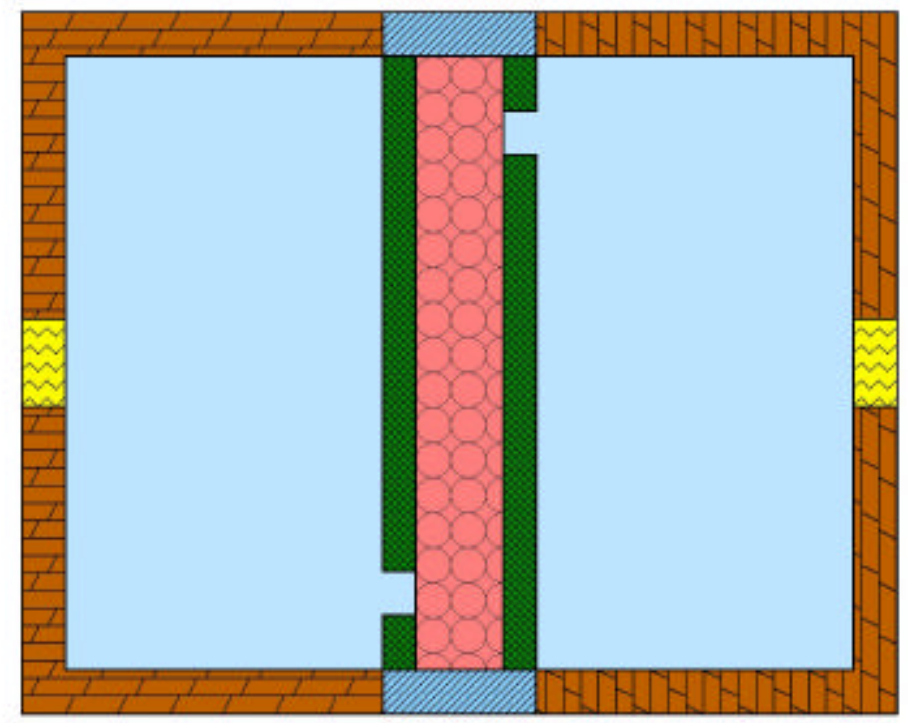

Wall 1 b.c.'s $(\mathrm{U}=0, \mathrm{~T}=\mathrm{T} 1)$

Wall 2 b.c.'s (U=0, T=T2)

Inlet or outlet b.c.'s (U, P, T)
Air (Navier-Stokes \& energy eqns.)

Wall sheathing (conduction eqn.)

Insulation (Darcy's Law \& energy eqn.)

\section{Adiabatic wall}

Figure 3.App: Schematic of a leaking building wall cross-section showing the boundary conditions and governing equations used in the CFD simulations. The live control volumes (those enclosed by the outer boundary) represent air, insulation, and the wall sheathing. They 
are governed by the equations as shown in the figure. The outer boundary is composed of a wall supplying wall boundary conditions (zero flow velocity and a different fixed temperature on each side of the leaking wall) with an inlet on one side of the leaking wall and an outlet on the other. The inlet and outlet supply thermal and pressure (or velocity) boundary conditions.

\section{App. 2.2: Thermal equilibrium in the wall insulation}

A fundamental assumption in using a single fluid dynamics equation (equation 8) to represent energy transport in the wall insulation is local thermal equilibrium between the fluid and solid phases (air and glass fibers) of the insulation. If the two phases are not in thermal equilibrium then two separate energy equations must be used, one for the fluid phase and one for the solid phase.

In this section, an analysis is performed using a representative system to determine if our air/insulation system is in thermal equilibrium. In the representative system air flows into a porous media (the insulation) at a velocity and temperature of $U$ and $T_{a}$, respectively. The solid part of the porous media (glass fibers) maintains a constant temperature of $T_{s}$. As air flows into the porous media it exchanges heat with glass fibers and the air temperature changes until it eventually reaches the glass fiber temperature. At this point, they are in thermal equilibrium. For this analysis, we will consider the fluid and solid to be in equilibrium when there is a temperature difference of 0.1 percent or less between the phases.

$$
\begin{gathered}
\rho c_{p} V \frac{d T}{d t}=h A_{s}\left(T_{s}-T\right) \\
N u_{D}=0.3+\frac{0.62 \operatorname{Re}^{1 / 2} \operatorname{Pr}^{1 / 3}}{\left[1+(0.4 / \operatorname{Pr})^{2 / 3}\right]^{1 / 4}} \\
h=N u_{D} k / D
\end{gathered}
$$

In this analysis, a control mass of air is followed as it flows through the porous media. The temperature of the air is calculated versus time, or correspondingly penetration distance into the porous media, via equation 4.App. Equation 4.App is merely a statement of the First Law of Thermodynamics; the control mass enthalpy time rate of change (lhs) is equal to the rate of heat transfer (rhs). In equation 4.App, $V$ is the control mass volume, $h$ is the heat transfer coefficient, $A_{s}$ is the fiber surface area, and $T$ is air temperature.

The heat transfer coefficient for cross-flow over a cylinder is used in these calculations as this flow geometry most closely resembles our case (the glass fibers are essentially circular cylinders). Equations 5.App and 6.App give the relations used to determine the heat transfer coefficient based on Reynolds number, Prandtl number, and fiber diameter $(D)$. The control mass volume is arbitrarily set to $1 \mathrm{~m}^{3}$. In our system, the insulation is 99 percent air by volume and 1 percent glass fibers. The fiber surface area is determined from the volume fraction and by setting the fiber diameter to 10 microns (a typical diameter for glass fiber insulation) and assuming the fibers are 1 meter in length. 
The time it takes incoming air to reach thermal equilibrium and the corresponding penetration distance into the porous media are calculated with equations 4.App to 6.App. The air temperature versus penetration distance into the porous media (insulation) is shown in figure 4.App. In this case, there is a nominal non-dimensional leakage rate $\left(a_{o}\right)$ of one (corresponding to a flow velocity of about $0.08 \mathrm{~m} / \mathrm{s}$ ) and the bulk air and glass fiber temperatures are 274 and 298 Kelvin, respectively. The figure shows that as the air flows deeper into the insulation its temperature increases and eventually reaches the glass temperature at a distance of about $1.5 \times 10^{-5} \mathrm{~m}$. The air reaches thermal equilibrium with the glass fibers very quickly. Compared to the smallest dimension of the wall insulation $(0.1 \mathrm{~m})$ this distance is negligible. Therefore, it is appropriate to use a single equation to represent the energy transport in the wall insulation.

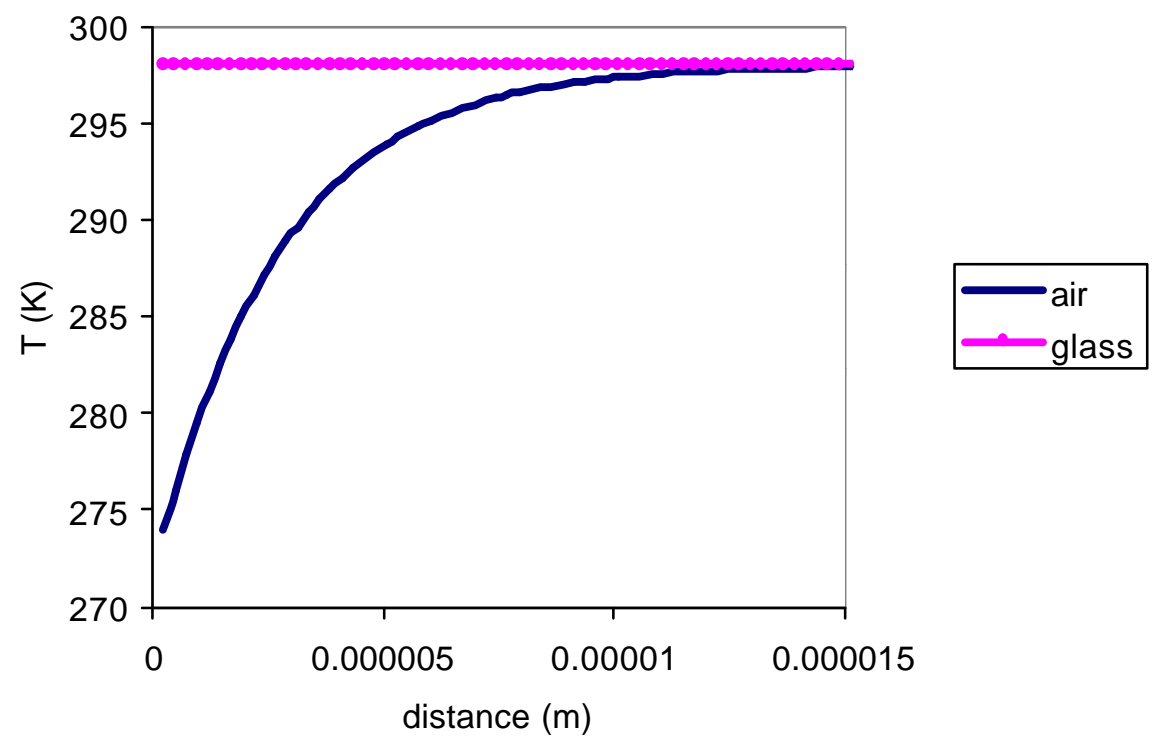

Figure 4.App: Air and glass fiber temperature versus penetration distance into the insulation. The glass fiber temperature is held constant at $298 \mathrm{~K}$ and the incoming air temperature is 274

$\mathrm{K}$. As the air flows deeper into the insulation its temperature increases and eventually reaches the glass fiber temperature at a distance of about $1.5 \times 10^{-5} \mathrm{~m}$.

Further calculations are performed to determine the equilibrium distance for the insulation at various flow rates and for another porous media, a packed bed composed of spherical glass balls ( $2 \mathrm{~cm}$ dia.). The non-dimensional flow rate is varied from about 0.25 to 2.25 to see how the equilibrium distance changes with flow velocity. Figure 5.App shows that the equilibrium distance increases almost linearly with flow rate. Note that the vertical scale showing distance is logarithmic. Even at the highest leakage rates (around 2 in most buildings) the equilibrium distance for the insulation would be very small compared to the wall dimensions, so a single equation for energy transport in the porous media would be appropriate. The situation is different for the packed bed, however. The equilibrium distance for the packed bed is roughly three orders of magnitude higher than that for the insulation. This is mainly due to the much lower surface area of the solid phase in the packed bed, which results in less heat transfer. In this case, the equilibrium distance is on the order of the wall 
thickness, so the air and solid phase would not have enough time to reach thermal equilibrium. Therefore, two equations would have to be used to represent energy transport in the packed bed: one for the solid phase and one for the fluid phase.

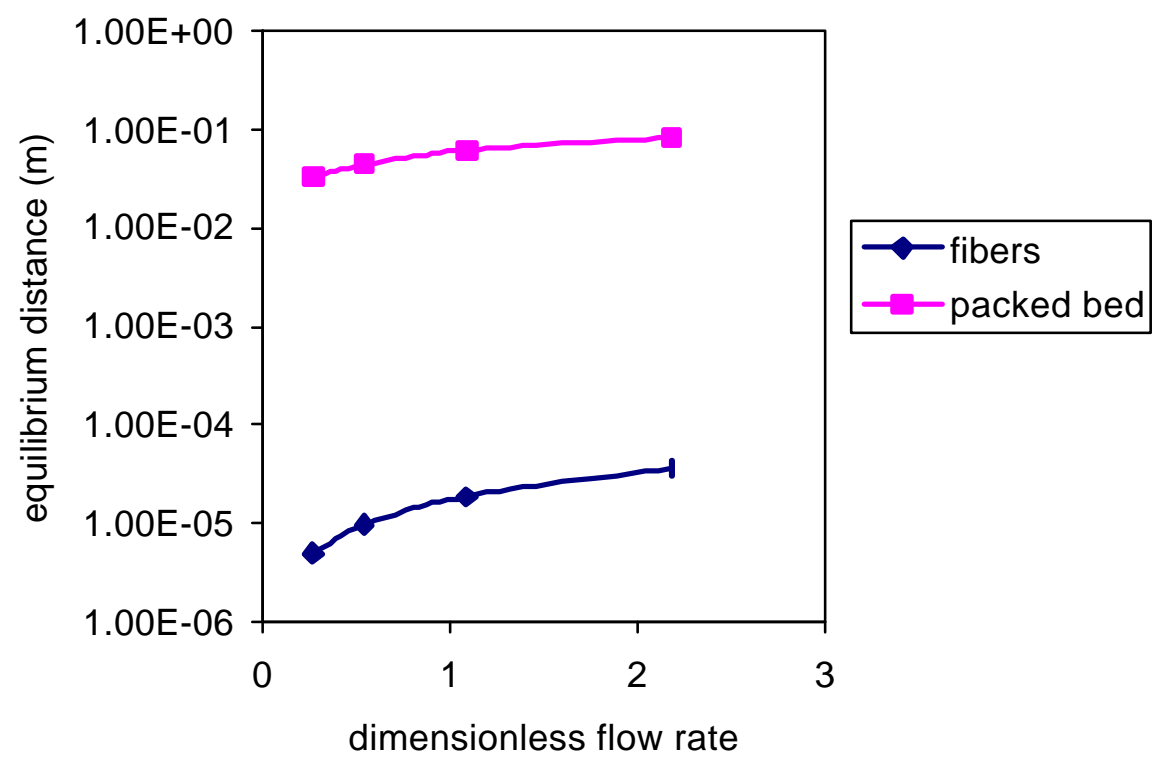

Figure 5.App: Equilibrium distance versus leakage rate for two different types of porous material: glass fibers and a packed bed of glass spheres. The glass fiber material (insulation) has a much shorter equilibrium distance than the packed bed suggesting that a single equation can be used for energy transport in the fibers, while two equations would have to be used for energy transport in the packed bed.

Finally, a macro-scale analysis is performed to determine how the glass fiber and air temperatures vary through the entire depth of a leaking wall. This is a one-dimensional analysis in which it is assumed that only the mode of heat transfer is from the glass fibers to air. In reality, this is not the case, but this analysis is good for illustrative purposes.

$$
\begin{array}{cl}
\rho c_{p} V A \frac{d T}{d x}=k A \frac{d^{2} T}{d x^{2}}+h A_{\text {surf }}\left(T_{s}-T\right) & \text { 7.App } \\
k_{s} A_{s} \frac{d^{2} T_{s}}{d x^{2}}-h A_{\text {surf }}\left(T_{s}-T\right)=0 & \text { 8.App }
\end{array}
$$

The governing equations for the air/fiber system are shown in equations 7.App and 8.App. Equation 7.App is the energy equation for air and 8.App is the energy equation for glass fibers. In the above equations, $V$ is the leaking air velocity, $A$ is the cross-sectional area of air, $h$ is the heat transfer coefficient (see equation 6.App), $A_{\text {surf }}$ is the glass fiber surface area (determined earlier in this section), $T_{s}$ is the fiber temperature, $T$ is the air temperature, $k_{s}$ is the glass thermal conductivity, $A_{s}$ is the total cross-sectional area of glass fibers, and $x$ is the distance into the wall. A simple analysis, in which the Peclet number $\left(P e=\rho V L c_{p} / k\right)$ is calculated using characteristic values, shows that convection is the dominant mechanism for 
energy transport in the air. This allows us to drop the conduction term in equation 7.App (second-order term) in this analysis.

The governing equations are solved using standard techniques of ODE's under the following boundary conditions: $T(0)=T_{\text {out }}, T_{s}(0)=T_{\text {out }}$, and $T_{s}(L)=T_{\text {in }}$, where $T_{\text {out }}$ is the outside temperature $(274 \mathrm{~K})$ and $T_{\text {in }}$ is the inside temperature $(298 \mathrm{~K})$. The standard solution for $T$ and $T_{s}$ is given below in equations 9.App and 10.App. In the equations, $C_{1}, C_{2}$, and $C_{3}$ are constants, $r_{1}, r_{2}$, and $r_{3}$ are roots of the auxiliary equation, and $\lambda$ is a ratio of certain problem parameters, i.e., $\rho V A c_{p} / h A_{\text {surf. }}$ The actual values for the constants, the roots, and $\lambda$ vary with the assigned parameter values in the equations. Figure 6.App shows the air and glass fiber temperatures through the wall for a given, characteristic set of parameter values. The two curves essentially overlap, as the temperatures are nearly equal. The same behavior occurrs with all realistic variations in the parameters, indicating that the assumption of thermal equilibrium between the air and glass fibers is valid.

$$
\begin{array}{lr}
T(x)=C_{1} e^{r_{1} x}+C_{2} e^{r_{2} x}+C_{3} e^{r_{3} x} & \text { 9.App } \\
T_{s}(x)=T+\lambda \frac{d T}{d x} & \text { 10.App }
\end{array}
$$

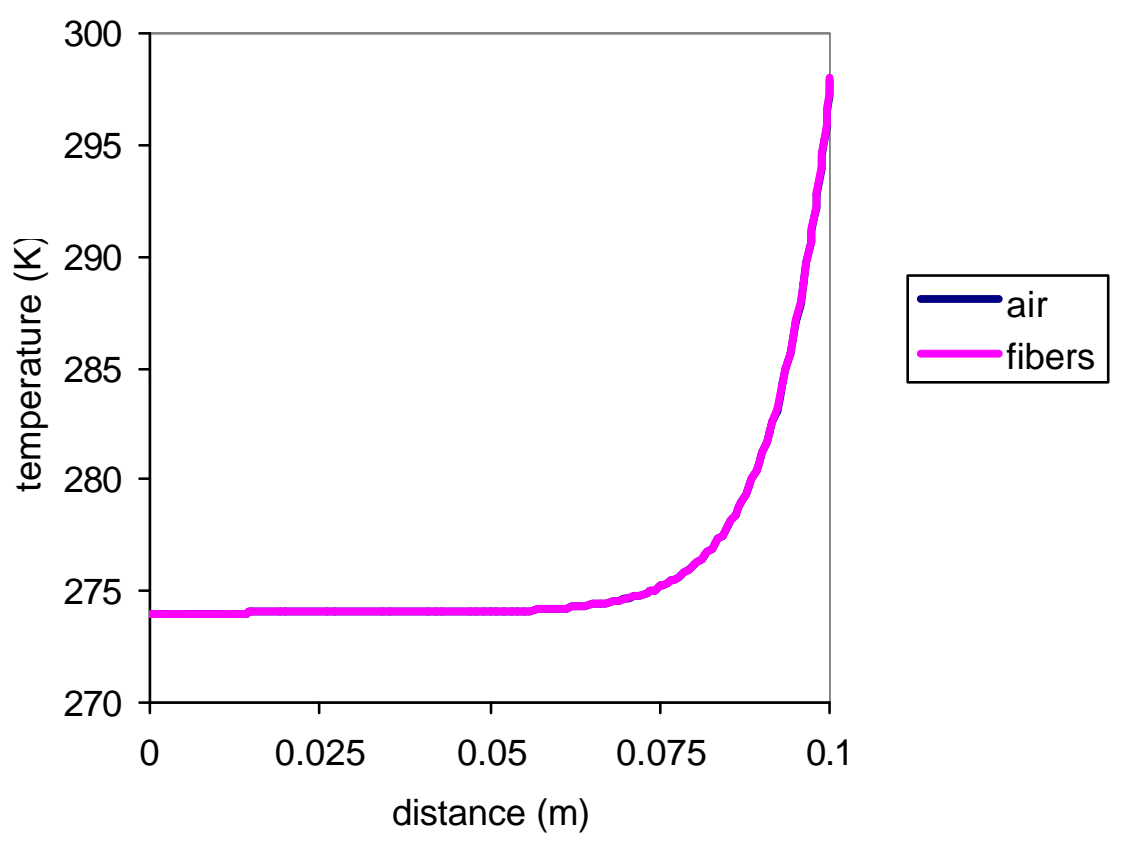

Figure 6.App: Air and glass fiber temperatures versus distance through the leaking wall for a chosen set of system parameters. There are two curves plotted, but only one is apparent because the air and fiber temperatures are nearly identical. This further supports the assumption of thermal equilibrium between the air and glass fibers. 


\section{App. 2.3: Solution Methodology}

Although we want steady-state values for our analysis, it was not possible to achieve a converged solution to the steady-state equations (i.e., no time dependent term). The solution either diverged or would not converge to the set limits when solving the steady-state equations. The convergence criteria are that the sum of the normalized residuals are less than or equal to than $10^{-3}$ for the continuity and momentum equations and $10^{-6}$ for the energy equation. These are the standard convergence criteria suggested in the Fluent user guides.

In an attempt to achieve a converged solution, the transient equations were solved and allowed to proceed in time until a steady-state solution was reached. This would have worked well, but it would have taken too long. Using a time-step of $0.1 \mathrm{sec}$ provided a converged solution at each time step and proceeded nicely toward steady-state, but it would have taken days to complete a single simulation. To speed up the solution process, a method was developed in which the time step was gradually increased from $0.1 \mathrm{sec}$ to $60 \mathrm{sec}$ and then gradually decreased again to $0.1 \mathrm{sec}$. The time step size was changed gradually because it was found that sudden changes in the time step could result in divergence of the solution. By increasing the time step to a large size of $60 \mathrm{sec}$, the flow and thermal fields can develop much more quickly (in wall clock time) which helps to reduce the overall simulation time. Unfortunately, with the $60 \mathrm{sec}$ time step the solution did not converge, so it was necessary to reduce the time step back down to $0.1 \mathrm{sec}$ to provide a converged solution for the final results. Since the solution process uses an iterative method, the final results at the end of the simulation are all that really matters. All of the previous values calculated during the solution process, whether converged or not, can be considered improved initial guesses in the iterative process that lead to the final solution.

One additional technique was used to speed up the solution process. At several points in the simulation, when the $60 \mathrm{sec}$ time step was in use, the solution was stopped and the equations were decoupled. The continuity and momentum equations were turned off (the flow field was frozen in time) and the energy equation was allowed to proceed in time until a steady-state solution (based on the intermediate frozen flow field) was reached. Then, the continuity and momentum equations were turned back on and the solution proceeded with the equations coupled. After a certain number of time steps have passed, the equations were decoupled again and the process repeated. This helped speed up the solution because the thermal field developed slowly, relative to the flow field, due to the insulating effects of the wall. In this way, the thermal field was allowed to jump ahead of the flow field and very quickly reach an intermediate solution. Then, once the flow equations were turned back on the flow and thermal fields would interact and come back into equilibrium.

This entire method is a fairly common technique for speeding up solutions of complex CFD problems and is discussed in the Fluent users guide (Fluent 4.4 user guide, Volume 3, Chapter 16, pages 44-49). The solution process used in this work is detailed below.

\section{Solution Process:}

1) Provide initial guess (initial conditions) for flow and thermal fields. All velocities are set to zero. The left half of the domain is set to the inside temperature (or outside depending on whether the wall is infiltrating or exfiltrating) and the right half is set to the outside temperature (or inside temp.)

2) The solution is begun with a $0.1 \mathrm{sec}$ time step and proceeds for 5 time steps. 
3) The time step size is changed to $1 \mathrm{sec}$ and the solution proceeds for another 5 time steps.

4) The time step size is changed to $10 \mathrm{sec}$ and the solution proceeds for another 5 time steps.

5) The time step size is changed to $60 \mathrm{sec}$ and the solution proceeds for another 60 time steps.

6) The flow equations are turned off and the energy equation is solved to steady-state using the intermediate flow field.

7) The flow equations are turned back on and the solution proceeds for another 60 time steps with a time step size of $60 \mathrm{sec}$.

8) Steps 6 and 7 are repeated twice.

9) The time step size is reduced to $10 \mathrm{sec}$ and the solution proceeds for 20 time steps.

10) The flow equations are turned off and the energy equation is solved to steady-state using the intermediate flow field.

11) The flow equations are turned back on and the solutions proceeds for another 20 time steps.

12) Steps 10 and 11 are repeated once.

13) The time step is reduced to $1 \mathrm{sec}$ and the solution proceeds for 60 time steps.

14) The flow equations are turned off and the energy equation is solved to steady-state using the intermediate flow field.

15) The flow equations are turned back on.

16) The time step is reduced to $0.1 \mathrm{sec}$ and the solution proceeds for 100 time steps.

17) The data is written to a file.

\section{Appendix 3: Sample CFD Results: Energy Flux through Leaking Walls}

In the framework of this study, a building envelope section with no air leakage has only one component in the energy flux through the wall, pure conduction, denoted as $Q_{o}$. An envelope section which experiences air leakage has two components in the energy flux, that due to conduction $\left(Q^{*}{ }_{o}\right)$ and that due to convection or infiltration $\left(Q_{i n f}\right)$. In calculation of the heat recovery factor, the non-leaking elements subtract out of the equation (see equation 2 ), so only the leaking walls need to be considered. Therefore, in this study a complete room is constructed by combining two leaking walls, one infiltrating and one exfiltrating. This is illustrated in figure 7.App.

The infiltrating and exfiltrating walls are simulated independently and the four flux terms (two for each wall) are added together to determine the heat recovery factor for a given leakage rate. In each case, the conduction and convection flux component is determined at the external (outside) face of the wall as shown in figure 7.App. 

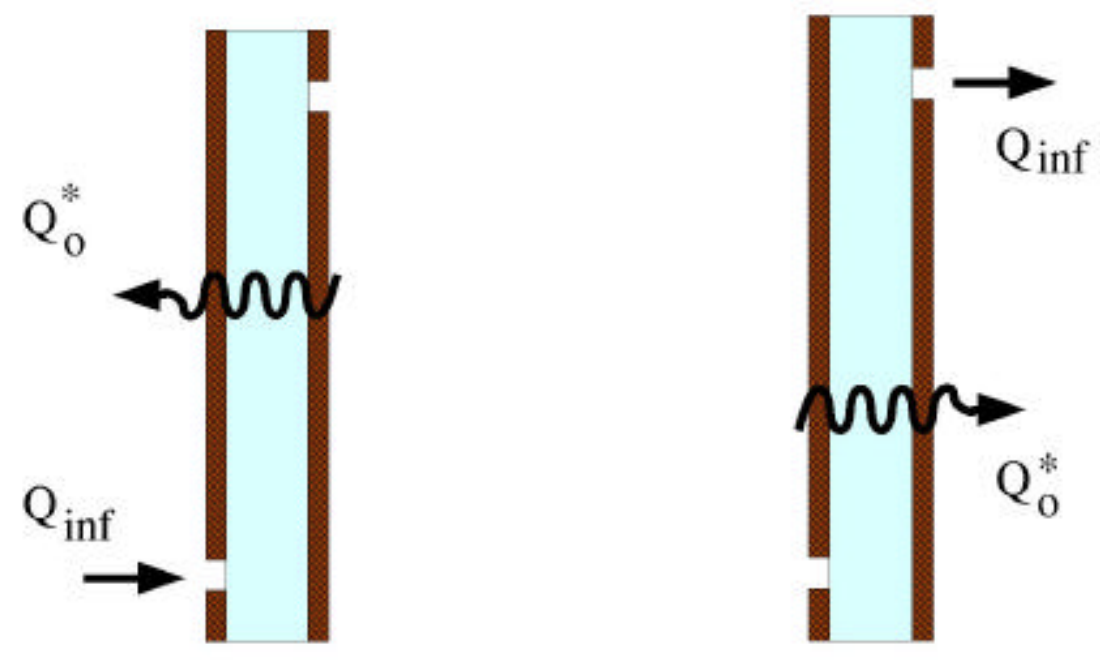

Figure 7.App: Convection $\left(Q_{i n f}\right)$ and conduction $\left(Q^{*}{ }_{o}\right)$ energy flux components through infiltrating (left) and exfiltrating (right) walls with a hi/low hole configuration (wall 1). The directions of leaking air (convection) and conduction are anti-parallel in the infiltrating case and parallel in the exfiltrating case. The control volume boundary is the external face of the walls, i.e., the left face of the infiltrating wall and right face of the exfiltrating wall.

As the leakage rate varies, the conduction and convection flux components change in both the infiltrating and exfiltrating walls. The total energy flux through the boundary of the room system changes, also. This is because leaking air alters the thermal profiles in and near the wall changing the overall heat transfer. This is illustrated in figure 8.App using CFD results from an infiltrating wall 1 configuration. Figure 8.App shows that parts of the wall that are far away from the holes in the sheathing (the middle section of the wall in this case) have a nearly linear temperature profile through the wall. This is similar to a wall with no leakage. Leaking air does not seem to have a significant effect in this region. In the vicinity of the holes, leaking air changes the thermal profiles in the wall and significantly impacts the heat transfer through this region.
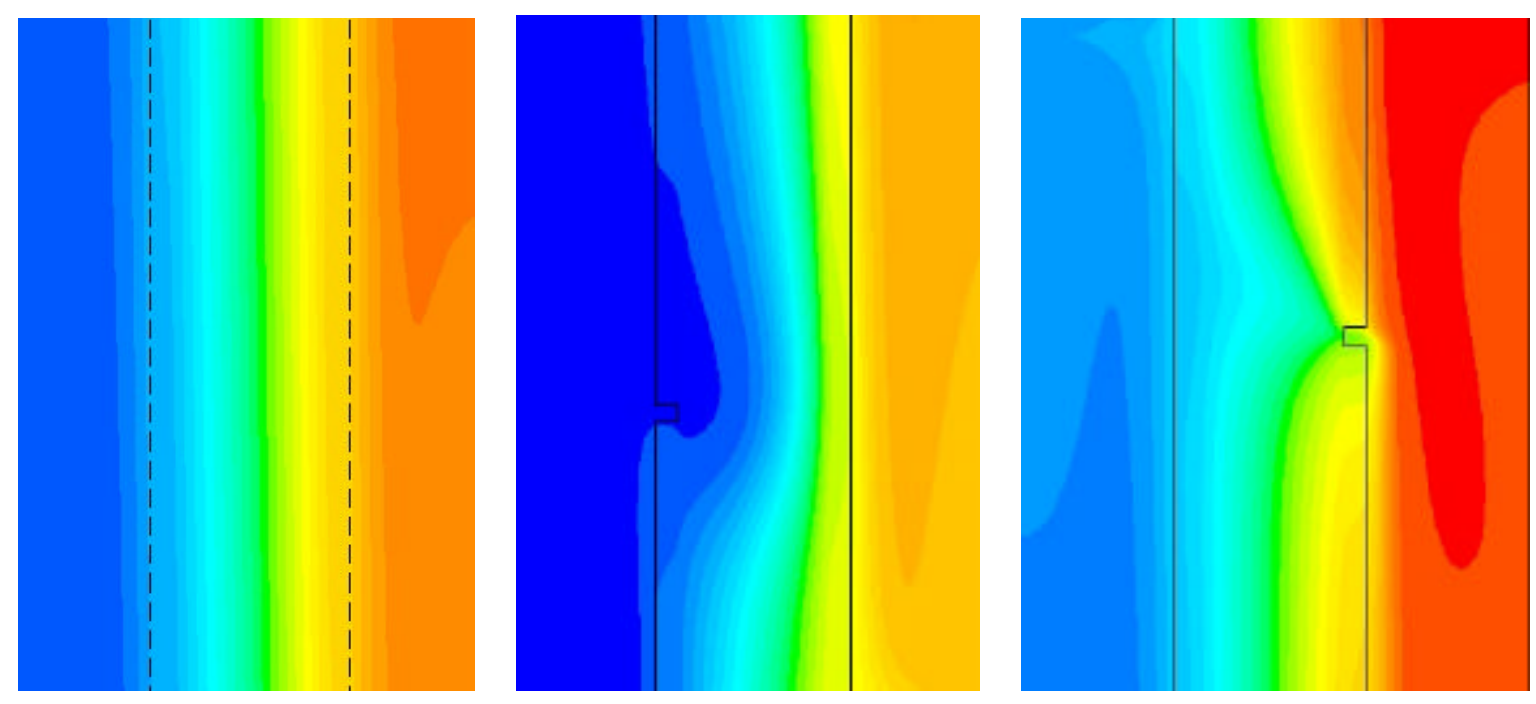
Figure 8.App: Thermal profiles at various locations of an infiltrating wall with a hi/low hole configuration (wall 1). The wall outline is represented by the black line, the outside temperature is cold (represented by blue), and the inside is warm (represented by red). The picture on the left shows thermal profiles in the middle section of the wall far away from holes in the sheathing. This section has essentially a linear temperature profile through the wall just as a wall with no leakage does. The profile here looks like that for a wall with pure conduction. The middle picture shows thermal profiles near the hole through which air leaks from outside into the wall. It is clear that the infiltrating air cools this region and significantly alters the thermal profile in the wall. The picture on the right shows thermal profiles near the hole through which leaking flows from the wall cavity into the room. The leaking air also alters the thermal profiles in this region.

The conduction and convection components for the insulated wall with a hi/low hole configuration (wall 1) are shown in figures 9.App and 10.App versus a non-dimensionalized form of the leakage rate $\left(a_{o}\right)$. Figure 9.App shows the flux components for an infiltrating wall. The conduction flux drops and the convection flux rises with increasing leakage rate. In this case, the flow direction of leaking air (into the room) is opposite the direction of conduction (out of the room)—see figures 1.App. and 7.App. Recall, the indoor temperature is greater than the outdoor temperature so energy conducts out of the room. Leaking air in the infiltrating wall opposes conduction, so as the leakage rate increases the conduction flux decreases. The convection flux scales almost linearly with the leakage rate.

The energy flux components for the exfiltrating wall are shown in figure 10.App. In this case, the flow direction of leaking air is the same as the direction of conduction, out of the room. Leaking air enhances the conduction flux and this component increases with leakage rate. Again, the convection flux scales almost linearly with the leakage rate.

These figures illustrate how the important physical mechanisms at work in a building wall experiencing air infiltration (conduction and convection) affect one another and how their relative contributions to the energy flux vary with leakage rate. During infiltration the two mechanisms oppose each another, and during exfiltration they enhance one another. This same behavior is seen in all leaking walls regardless of the construction details or the leakage path geometry. It is the coupled nature of the mechanisms that give rise to the phenomena of infiltration heat recovery. The heat recovery, however, is masked in the numbers and can only be determined by adding together the flux components from both (all) of the walls and performing the proper calculations. 


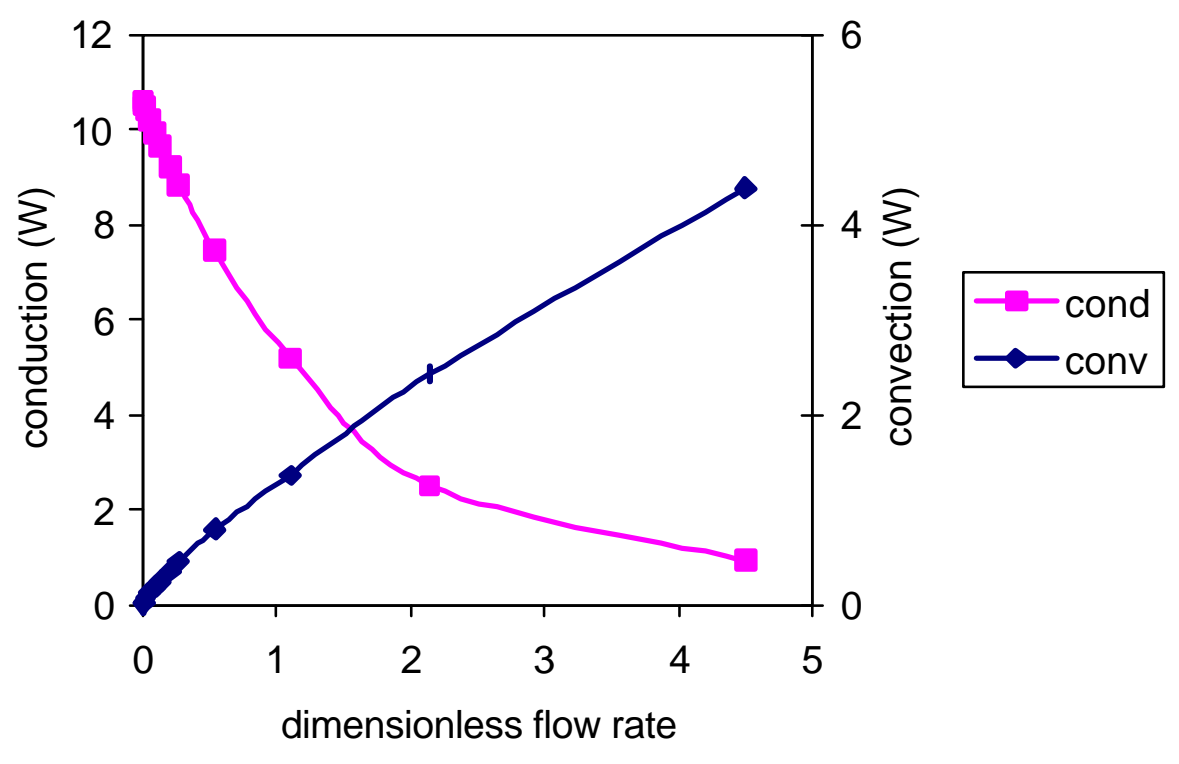

Figure 9.App: Convection and conduction energy flux components through an infiltrating wall with a hi/low hole configuration (wall 1). Leaking air opposes conduction, in this case, so this component drops with increasing leakage rate. The convection component increases almost linearly with leakage rate.

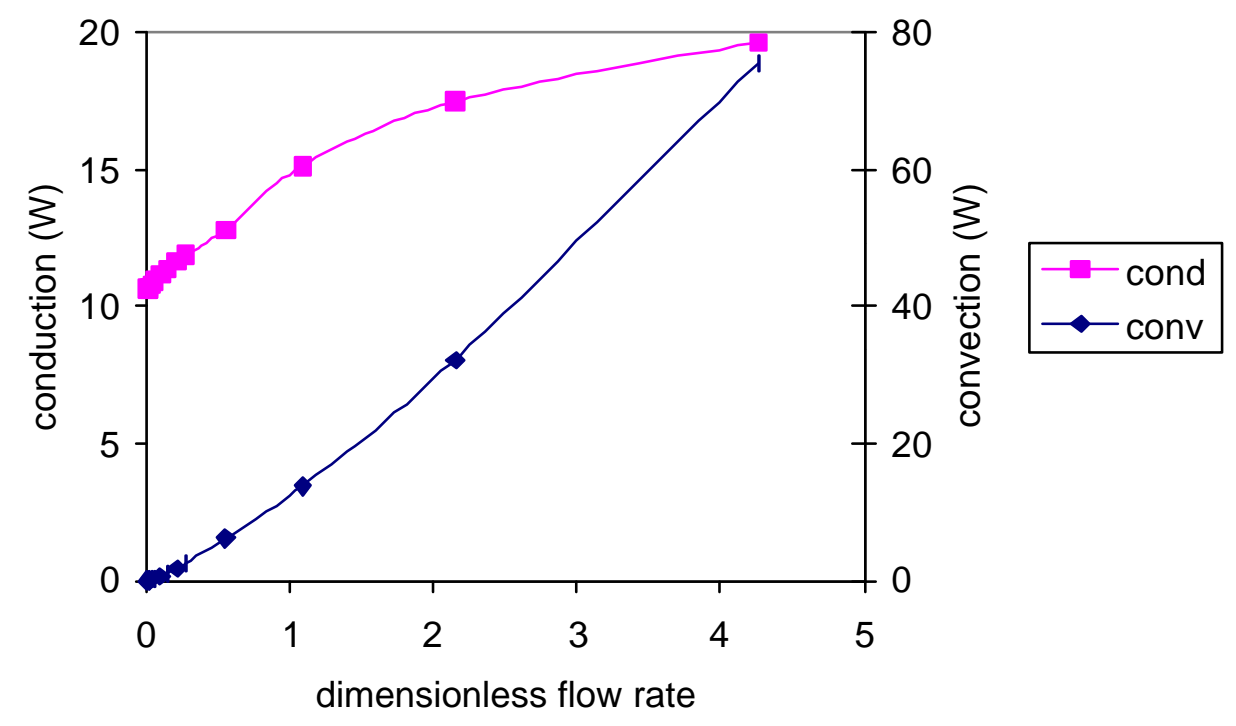

Figure 10.App: Convection and conduction energy flux components through an exfiltrating wall with a hi/low hole configuration (wall 1). Leaking air enhances conduction, in this case, so this component grows with increasing leakage rate. The convection component increases almost linearly with leakage rate. 


\section{Appendix 4: A Worked Example Using the Infiltration Heat Recovery Factor}

In this section, sample calculations are performed to illustrate the impact of infiltration heat recovery on building energy load predictions. Heat recovery values from CFD simulations of the wall 1 geometry (see figure 4) are used. Infiltration and total energy loads are calculated using the conventional method and an improved method which accounts for heat recovery over the range of leakage rates to provide an idea of the error incurred by not considering infiltration heat recovery.

In these calculations, a nominal value of $3000 \mathrm{~W}$ is used for the building conduction load $\left(Q_{o}\right)$. This should be reasonable for a moderate-sized residential building in fairly cold weather. The infiltration ( $Q_{\text {infC }}$ and $\left.Q_{\text {inf }}\right)$ and total $\left(Q_{C}\right.$ and $\left.Q\right)$ energy loads are calculated via equations 1.App and 3.App.

The conventional and actual infiltration loads are plotted versus the nondimensionalized flow rate in figure 11.App. The conventional load estimate increases linearly with flow rate as it is merely the product of the leakage rate, the specific heat of air, and the bulk air temperature difference. The actual energy load is significantly less than the conventional estimate and increases in a non-linear fashion. This plot shows that heat recovery acts to reduce the infiltration load.

The total building energy loads, conventional and actual, are shown in figure 12.App. Again, the actual load is substantially lower than the conventional load. The difference between the two values is due to heat recovery. The size of the difference is shown in figure 13.App. This figure shows that the difference between the conventional total load and the actual total load is small at low leakage rates and grows with increasing leakage rate reaching a maximum difference of about 25 percent in this case. At high leakage rates the error begins to decrease because the heat recovery is small. The percent difference indicates the error that would be incurred in total building energy load predictions that do not include infiltration heat recovery. 


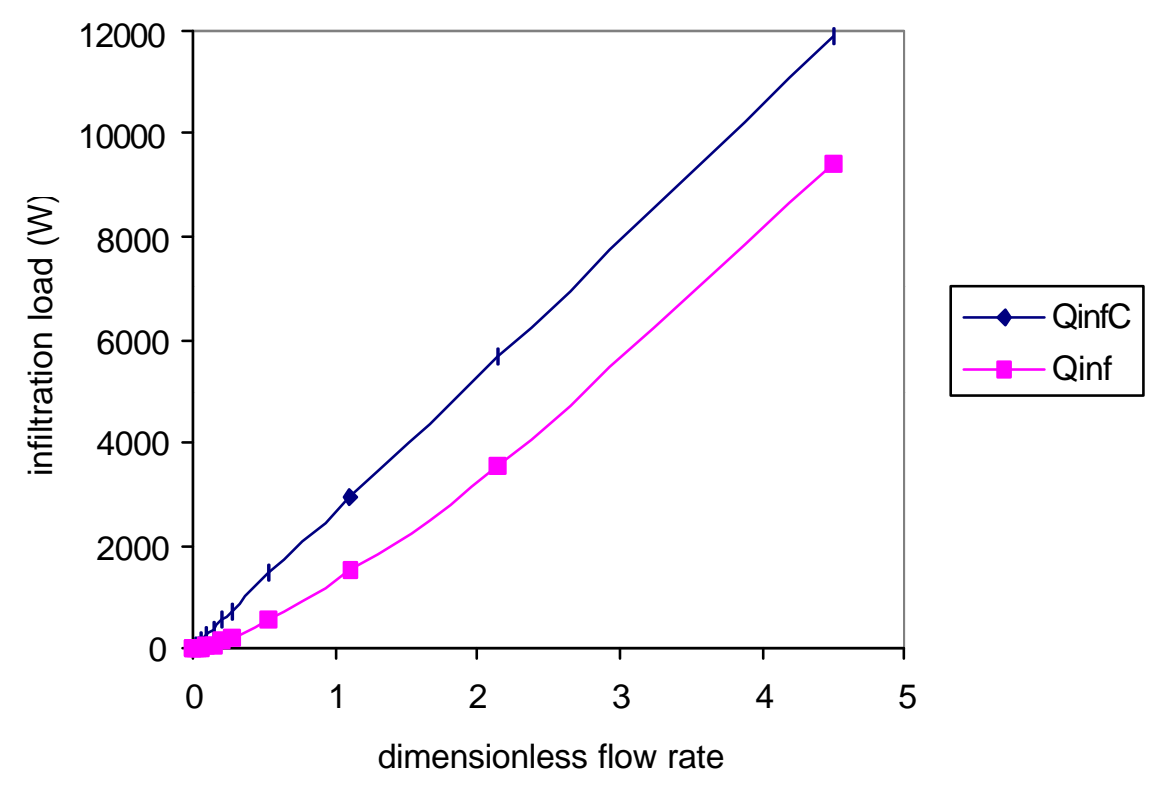

Figure 11.App: Infiltration energy loads for the wall with a hi/low hole configuration (wall 1). $Q_{\text {inf } C}$ is the conventional infiltration load, calculated as $m c_{p} \Delta T$, and does not account for heat recovery. $Q_{i n f}$ is the actual infiltration load, calculated as (1-E) $Q_{i n f C}$, and accounts for heat recovery through the heat recovery factor $\varepsilon$. The actual infiltration load is always less than the conventional estimate because of heat recovery.

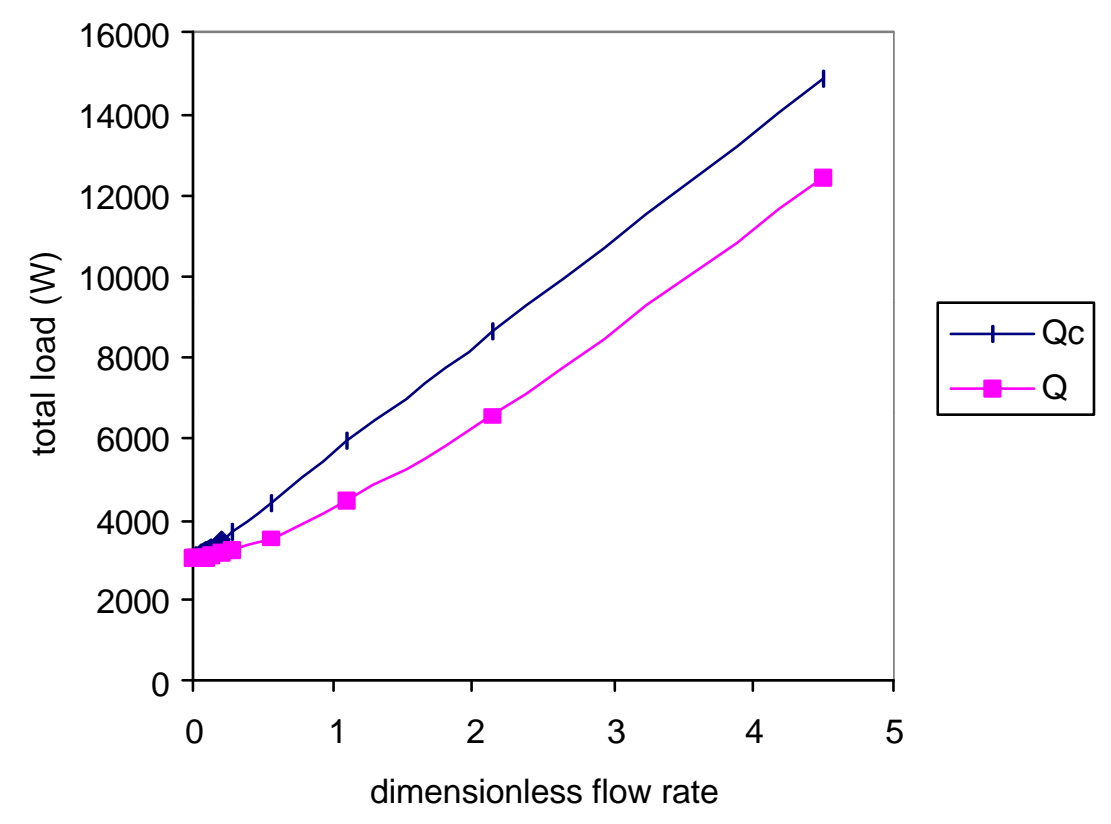

Figure 12.App: Total energy loads (conduction plus infiltration) for the wall with a hi/low hole configuration (wall 1). $Q_{C}$ is the conventional total load and $Q$ is the actual total load. A constant set value of $3000 \mathrm{~W}$ is used for the conduction load. Note that the actual total load is always less than the conventional estimate because of infiltration heat recovery. 


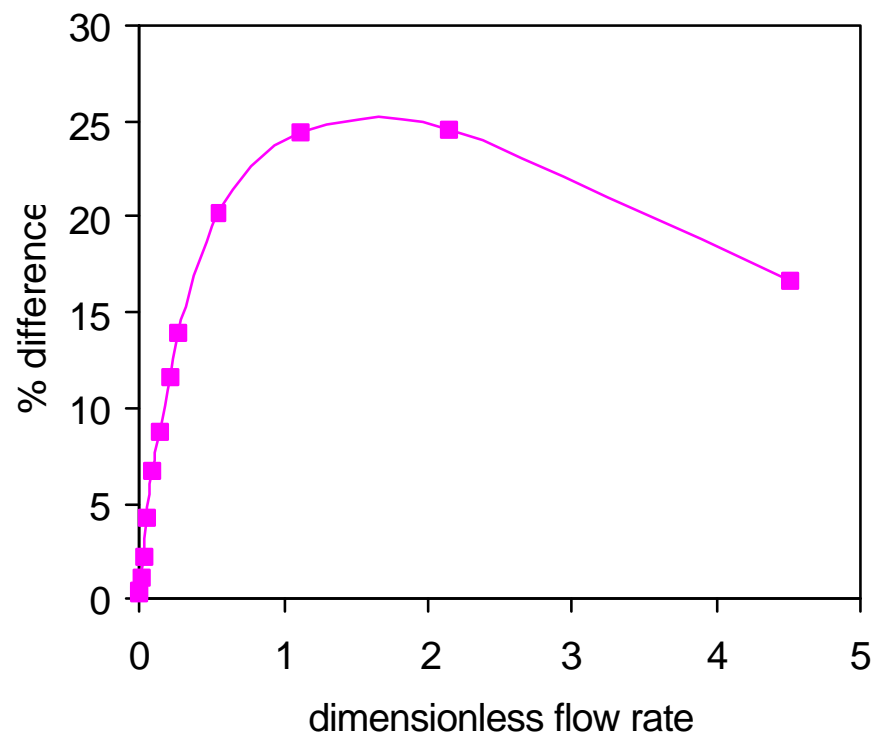

Figure 13.App: Percent difference between the conventional and actual total energy load. This is the error incurred by using the conventional estimate for the infiltration load. 